\title{
A Decision-Making Approach for the Evaluation of Information Security Management under Complex Intuitionistic Fuzzy Set Environment
}

\author{
Muhammad Azam, ${ }^{1}$ Muhammad Sajjad Ali Khan $\mathbb{D}^{2},{ }^{2}$ and Shilin Yang ${ }^{1}$ \\ ${ }^{1}$ College of Applied Science, Beijing University of Technology, Beijing 100022, China \\ ${ }^{2}$ Department of Mathematics, Khushal Khan Khattak University, Karak, KP, Pakistan \\ Correspondence should be addressed to Muhammad Sajjad Ali Khan; sajjadalimath@yahoo.com
}

Received 3 May 2021; Revised 22 June 2021; Accepted 13 December 2021; Published 21 February 2022

Academic Editor: Musavarah Sarwar

Copyright (c) 2022 Muhammad Azam et al. This is an open access article distributed under the Creative Commons Attribution License, which permits unrestricted use, distribution, and reproduction in any medium, provided the original work is properly cited.

\begin{abstract}
Since intuitionistic fuzzy set only deals with uncertainty but not periodicity, therefore to overcome, this situation complex intuitionistic fuzzy set is a better tool that deals with both uncertainty and periodicity. Also, Bonferroni mean operator has the advantage of considering interrelationships between parameters, but it deals with only the crisp data. Recently, to deal with fuzzy data, many extensions of Bonferroni operators have been developed. Motivated by the CIFS and BM operators, in this paper, we proposed some Dombi Bonferroni mean operators to deal with CIF information. Dombi Bonferroni mean operators are special cases of general T-conorm and T-norm, which have the advantage of good flexibility with a general parameter. We proposed the complex intuitionistic fuzzy Dombi Bonferroni mean (CIFDBM) operator, complex intuitionistic fuzzy Dombi weighted Bonferroni mean (CIFDWBM) operator, complex intuitionistic fuzzy Dombi geometric Bonferroni mean (CIFDGBM) operator, and complex intuitionistic fuzzy Dombi weighted geometric Bonferroni mean (CIFDWGBM) operator. Some properties of the developed operators are discussed in detail, and different cases are investigated. Moreover, a multicriteria group decision-making (MCGDM) method is developed based on the proposed aggregation operators. Finally, a numerical example of information security management evaluation is given in order to demonstrate the application and effectiveness of the proposed approach. A comparative study is also conducted in order to show the advantage of the developed method.
\end{abstract}

\section{Introduction}

The multicriteria group decision-making (MCGDM) approach is one of the methods for selecting the best option from a collection of alternatives. We come across different forms of decision-making (DM) problems in our everyday lives. Therefore, in order to solve such problems, we need to learn how decisions are made. Typically, the DM method requires crisp information, but complexity plays an important role in any DM problem in many everyday practical problems, and as a result, the information may not be in the form of a crisp collection. The notion of the fuzzy set (FS) was therefore developed by Zadeh [1] to deal with these situations and is characterized by a membership degree (MD) belonging to the closed interval $[0,1]$. However, the fuzzy set only addresses the level of satisfaction or dissatisfaction with the DM problem. Therefore, to overcome this situation, Atanassov [2] proposed the idea of intuitionistic fuzzy set (IFS), which combines both qualities by adding the non-membership degree to the FS and satisfying the condition that the number of the membership degrees is less than or equal to 1 . Several scholars and authors have presented their theories and methodologies [3-7] to explore the problems of DM and extend them to different disciplines under this theory. Song et al. [8] developed a similarity measure for IFSs and applied the definition to a medical diagnosis problem. Furthermore, Liu et al. [9] proposed some new operators based on Dombi operational laws [10] and Bonferroni mean (BM) operators [11], such as intuitionistic fuzzy Dombi Bonferroni mean (IFDBM) operators, 
to deal with the multicriteria group decision-making (MCGDM) problem. Khan et al. [12] presented prioritized aggregation operators for Pythagorean fuzzy information, and also Khan et al. [13] developed Einstein T-norm and Tconorm-based operators to deal with MCDM problems under the PF environment. Khan et al. $[14,15]$ developed interval-valued Pythagorean fuzzy Choquet integral operators. However, the PFN has also some restrictions on the scope of information. Khan et al. [16] introduced the notion of Pythagorean hesitant fuzzy sets and proposed decisionmaking approaches based on the Pythagorean hesitant fuzzy TOPSIS method [17] and Pythagorean hesitant fuzzy Choquet integral [18] to solve MCGDM problems. Moreover, Garg and Rani [19] proposed a new decision-making technique based on a hesitant fuzzy set. Batool et al. [20] proposed an MCDM technique for the fog-haze factor assessment problem under a Pythagorean probabilistic hesitant fuzzy environment. Based on maximizing deviation and the TOPSIS method, Garg and Rani [21] developed a MADM method under a simplified neutrosophic hesitant fuzzy environment. Khan et al. [22] developed a new ranking method for q-Rung Orthopair fuzzy values and proposed a new graphical ranking method based on hesitancy index and entropy. Khan et al. [23] introduced a linguistic q-Rung Orthopair fuzzy set and applied the notion to decisionmaking problems. As discussed, that these theories have their own advantages, however, the theory of soft sets introduced by Molodtsov [24] carried out a shovel work as it generalizes all the theories. In 2000, Lee [25] introduced another generalization of these theories in the form of bipolar-value fuzzy sets. Recently, Mahmood [26] introduced a new type of bipolar soft set called "T-bipolar soft set" and showed that the new approach is closer to the concept of bipolarity as compared to the previous ones.

Researchers have considered the MCGDM problems with FS and IFS, which are only able to deal with uncertainty and vagueness that occurs in results, according to the above discussion. These models are unable to account for the data's partial effect when dealing with data and its uncertainty at a particular point in time. However, in complex datasets, uncertainty and vagueness in the data occur simultaneously with changes in the data's process. To address these issues, Romot et al. [27] proposed the concept of a complex fuzzy set (CFS), which is defined as a complexvalued membership degree with a codomain unit disc in a complex plane. Many researchers [28-30] have conducted research in the area of CFS. However, CFS only models the agreement of elements in any set and does not discuss the disagreement. Thus, to handle such situations, Alkouri and Salleh [31] proposed the concept of a complex intuitionistic fuzzy set (CIFS), an extension of CFS, and is characterized by a complex-valued membership degree. In [32], the authors discussed the relation, projection, composition between CIFSs. They also developed distance measure for CIFS and proposed some operational laws for CIFS. To solve an MCDM problem, Rani and Garg [33, 34] developed some T-norm and T-conorm-based generalized averaging operators, power operators, and distance measures, respectively.
Garg and Rani [21] developed a variety of similarity measures and entropy measures for CIFS; they discussed some properties and applied the concept to MCDM problems and extended the concept of Bonferroni mean [11] operators. Garg and Rani [19] proposed some complex intuitionistic fuzzy Bonferroni mean operators with their different properties and applied the concept to the MCGDM problem. However, Bonferroni mean cannot be processed by Dombi operations. In addition, because CIFNs can describe fuzzy information easier, it is an increasing demand to combine the BM operator and the Dombi operations to deal with CIFNs for dealing with MCGDM problems. The goal of this paper is to propose some new complex intuitionistic fuzzy Dombi Bonferroni mean (CIFDBM) operators for dealing with MCGDM problems with complex intuitionistic fuzzy information. CIFDBM operators not only discuss interrelationships among criteria but are also flexible to deal with MCGDM problem. Motivated by these, the main objectives of this article are as follows:

(a) Some new complex intuitionistic fuzzy Dombi Bonferroni mean (CIFDBM) operators with complex intuitionistic fuzzy setting are introduced in order to project time-periodic problems and twodimensional information simultaneously in one set.

(b) Some properties of the proposed operators are discussed in detail.

(c) A MCGDM method is proposed under the CIF environment. The applicability of the proposed method is explored on a real-life decision-making problem related to the evaluation of information security management. Finally, the proposed method is compared with existing methods in order to demonstrate the application and validity of the proposed approach.

The remaining of the paper is arranged as follows. Some basis definitions, operational laws, and aggregation operators are presented in Section 2. Section 3 deals with the complex intuitionistic fuzzy Dombi Bonferroni mean (CIFDBM) operator, complex intuitionistic fuzzy Dombi weighted Bonferroni mean (CIFDWBM) operator, complex intuitionistic fuzzy Dombi geometric Bonferroni mean (CIFDGBM) operator, and complex intuitionistic fuzzy Dombi weighted geometric Bonferroni mean (CIFDWGBM) operator. Some properties of the proposed operators are investigated, and various cases are discussed in detail. A MCGDM approach is proposed in Section 4. In Section 5, a numerical example for firewall selection problem is presented, and comparative study is also conducted. Concluding remark is presented in Section 6 .

\section{Preliminaries}

This section comprises some basic definitions and operational laws for complex intuitionistic fuzzy numbers.

\subsection{Complex Intuitionistic Fuzzy Set and Their Operations.} Alkouri and Salleh [30] first suggested the theory of complex intuitionistic fuzzy set as a generalization of the complex 
fuzzy set and intuitionistic fuzzy set. It is characterized by a complex-valued MD and complex-valued NMD.

Definition 1 (see [30]). Let $\tilde{\mathrm{A}}=\left\{a_{1}, a_{2}, \ldots, a_{n}\right\}$ be a universal set. Then, a complex intuitionistic fuzzy set can be defined by

$$
\mathscr{H}=\left\{a_{n},\left\langle\mathrm{M}_{\mathscr{H}}\left(a_{n}\right), \mathrm{N}_{\mathscr{H}}\left(a_{n}\right)\right\rangle \mid a_{n} \in \tilde{\mathrm{A}}\right\},
$$

where $\mathrm{M}_{\mathscr{H}}\left(a_{n}\right), \mathrm{N}_{\mathscr{H}}\left(a_{n}\right): \tilde{\mathrm{A}}=\{\vartheta: \vartheta \in \mathscr{H},|\vartheta| \leq 1\}$, defined by $\mathrm{M}_{\mathscr{H}}\left(\dot{\alpha}_{n}\right)=\Upsilon\left(a_{n}\right) e^{i 2 \eta_{\left(a_{n}\right)}}$ and $\mathrm{N}_{\mathscr{H}}\left(a_{n}\right)=\Lambda\left(a_{n}\right) e^{i 2 \lambda_{\left(a_{n}\right)} \text {, }}$ where $0 \leq \Upsilon\left(a_{n}\right), \eta, \Lambda\left(a_{n}\right), \lambda\left(a_{n}\right) \leq 1$ and $0 \leq \Upsilon\left(a_{n}\right)+$ $\Lambda\left(a_{n}\right), \eta\left(a_{n}\right)+\lambda\left(a_{n}\right) \leq 1$. A CIFN is denoted by $\mathscr{L}=\langle\langle\Upsilon, \eta\rangle,\langle\Lambda, \lambda\rangle\rangle$ such that $0 \leq \Upsilon+\Lambda, \eta+\lambda \leq 1$.

For two CIFNs $\mathscr{L}_{u}=\left\langle\left\langle\Upsilon_{u}, \eta_{u}\right\rangle,\left\langle\Lambda_{u}, \lambda_{u}\right\rangle\right\rangle(u=1,2)$, Alkouri and Salleh [23] defined a relation between them as

(1) $\mathscr{L}_{1} \subseteq \mathscr{L}_{2}$ if $\Upsilon_{\mathscr{L}_{1}} \leq \Upsilon_{\mathscr{L}_{2}}, \Lambda_{\mathscr{L}_{1}} \geq \Lambda_{\mathscr{L}_{2}}, \eta_{\mathscr{L}_{1}} \leq \eta_{\mathscr{L}_{2}}$, and $\lambda_{\mathscr{L}_{1}} \geq \lambda_{\mathscr{L}_{2}}$

(2) $\mathscr{L}_{1}=\mathscr{L}_{2}$ if and only if $\mathscr{L}_{1} \subseteq \mathscr{L}_{2}$ and $\mathscr{L}_{1} \supseteq \mathscr{L}_{2}$

(3) $\mathscr{L}_{1}^{c}=\left\langle\left\langle\Lambda_{u}, \lambda_{u}\right\rangle,\left\langle\Upsilon_{u}, \eta_{u}\right\rangle\right\rangle$

Garg and Rani [33] developed a score and accuracy function in order to compare two CIFNs. These can be defined by the following definition:

Definition 2 (see [33]). Let $\mathscr{L}=\langle\langle\Upsilon, \eta\rangle,\langle\Lambda, \lambda\rangle\rangle$ be CIFNs. Then, the score and accuracy function for CIFNs are defined as

$$
\mathcal{S}(\mathscr{L})=(\Upsilon-\Lambda)+(\eta-\lambda)
$$

and

$$
\mathscr{A}(\mathscr{L})=(\Upsilon+\Lambda)+(\eta+\lambda),
$$

respectively. Moreover, if $\mathscr{L}_{u}=\left\langle\left\langle\Upsilon_{u}, \eta_{u}\right\rangle,\left\langle\Lambda_{u}, \lambda_{u}\right\rangle\right\rangle$ $(u=1,2)$ are CIFNs, then $\mathscr{L}_{2} \leq \mathscr{L}_{1}$, if either $\left(\mathcal{S} \mathscr{L}_{2}\right) \leq \mathcal{S}\left(\mathscr{L}_{1}\right)$ or $\mathcal{S}\left(\mathscr{A} \ell_{1}\right)=\mathcal{S}\left(\mathscr{A} \ell_{2}\right)$, and $\mathcal{S}\left(\mathscr{A} \ell_{2}\right) \leq$ $\mathcal{S}\left(\mathscr{A}_{2}\right)$.

Some basic operational laws for CIFNs were proposed by Garg and Rani [33].

Definition 3 (see [33]). Let $\mathscr{L}_{j}=\left\langle\left\langle\Upsilon_{j}, \eta_{j}\right\rangle,\left\langle\Lambda_{j}, \lambda_{j}\right\rangle\right\rangle$ $(j=1,2)$ be CIFNs, and $\mathscr{E} \geq 0$ be any real scalar. Then, the algebraic operational laws for CIFNs are define as

(1) $\mathscr{L}_{1} \oplus \mathscr{L}_{2}=\left\langle\left\langle 1-(1-\Upsilon)_{1}(1-\Upsilon)_{2}\right), 1-\left(1-\eta_{1}\right)(1\right.$ $\left.\left.\left.-\eta_{2}\right)\right\rangle,\left\langle\Lambda_{1} \Lambda_{2}, \lambda_{1} \lambda_{2}\right\rangle\right\rangle$

(2) $\mathscr{L}_{1} \otimes \mathscr{L}_{2}=\left\langle\left\langle\Upsilon_{1} \Upsilon_{2}, \eta_{1} \eta_{2}\right\rangle,\left\langle\left(1-1-\Lambda_{1}\right)\left(1-\Lambda_{2}\right), 1-\right.\right.$ $\left.\left.\left(1-\lambda_{1}\right)\left(1-\lambda_{2}\right)\right\rangle\right\rangle$

(3) $\mathscr{E} \mathscr{L}_{1}=\left\langle\left\langle 1-\left(1-\Upsilon_{1}\right)^{\mathscr{E}}, 1-\left(1-\eta_{1}\right)^{\mathscr{E}}\right\rangle,\left\langle\Lambda_{1}^{\mathscr{E}}, \lambda_{1}^{\mathscr{E}}\right\rangle\right\rangle$

(4) $\mathscr{L}_{1 \mathscr{E}}^{\mathscr{E}}=\left\langle\left\langle\Upsilon_{1}^{\mathscr{E}}, \eta_{1}^{\mathscr{E}}\right\rangle, \quad\left\langle\left((1-(1-\Lambda))_{1}\right)^{\mathscr{E}},(1-1-\right.\right.$ $\left.\left.\left.\lambda_{1}\right)^{\mathscr{E}}\right\rangle\right\rangle$

In literature [21], Garg and Rani introduced different entropy measures and applied the concept to the MCDM problem. The CIF entropy measure can be defined as follows:
Definition 4 (see [21]). For any set $\mathscr{H} \in \psi(\tilde{\mathrm{A}})$, entropy measure $\hat{\mathrm{E}}: \psi(\AA) \longrightarrow[0,1]$ is a real-valued function satisfying the following properties:

(1) $0 \leq \hat{\mathrm{E}}(\mathscr{H}) \leq 1$

(2) $\hat{\mathrm{E}}(\mathscr{H})=0$, if $\mathscr{H}$ is a crisp set

(3) $\hat{\mathrm{E}}(\mathscr{H})=1 \Longleftrightarrow \Upsilon_{n}=\Lambda_{n}, \eta_{n}=\lambda_{n}$

(4) $\hat{\mathrm{E}}(\mathscr{H})=\hat{\mathrm{E}}\left(\mathscr{H}^{\mathrm{c}}\right)$

(5) $\hat{\mathrm{E}}(\mathscr{H}) \leq \hat{\mathrm{E}}(\mathscr{K})$ if either $\mathscr{H} \subseteq \mathscr{K}$ with $\Upsilon_{\mathscr{K}} \leq \Lambda_{\mathscr{K}}$ and $\eta_{\mathscr{H}} \leq \lambda_{\mathscr{H}}$ or $\mathscr{H} \supseteq \mathscr{K}$ with $\Upsilon_{\mathscr{K}} \geq \Lambda_{\mathscr{K}}$ and $\eta_{\mathscr{H}} \geq \lambda_{\mathscr{H}}$

Definition 5 (see [21]). Let $\mathscr{H}=\left\{a_{n},\left\langle\left\langle\Upsilon\left(a_{n}\right), \eta\left(a_{n}\right)\right\rangle\right.\right.$, $\left.\left.\left\langle\Lambda\left(a_{n}\right), \lambda\left(a_{n}\right)\right\rangle\right\rangle \mid a \in \tilde{\mathrm{A}}\right\}$ be a CIFS. Then, the entropy measure can be defined by

$$
\hat{\mathrm{E}}(\mathscr{H})=1-\frac{\sum_{\mathrm{j}=1}^{\mathrm{n}}\left(\left|\Upsilon\left(\mathrm{a}_{n}\right)-\Lambda\left(\mathrm{a}_{n}\right)\right|+(1 / 2 \pi)\left|\eta\left(\mathrm{a}_{n}\right)-\lambda\left(\mathrm{a}_{n}\right)\right|\right)}{\sum_{\mathrm{j}=1}^{\mathrm{n}}\left(\left|\Upsilon\left(\mathrm{a}_{n}\right)+\Lambda\left(\mathrm{a}_{n}\right)\right|+(1 / 2 \pi)\left|\eta\left(\mathrm{a}_{n}\right)+\lambda\left(\mathrm{a}_{n}\right)\right|\right)} .
$$

As we have discussed in the previous section, the BM operator is one of the better tools to deal with a DM problem. The BM operators not only deal interrelationships among criteria but are also more flexible. Therefore, Garg and Rani [19] proposed CIFBM operators as

Definition 6 (see [19]). Let $\mathscr{L}_{u}=\left\langle\left\langle\Upsilon_{u}, \eta_{u}\right\rangle,\left\langle\Lambda_{u}, \lambda_{u}\right\rangle\right\rangle$ $(u=1,2, \ldots, n)$ be CIFNs, $p, q \geq 0, k>0$. Then, a mapping CIFBM $^{p, q}: \mathscr{L}^{n} \longrightarrow \mathscr{L}$ is called CIFBM operator if

$$
\begin{aligned}
& \operatorname{CIFBM}^{p, q}\left(\mathscr{L}_{1}, \mathscr{L}_{2}, \ldots, \mathscr{L}_{n}\right) \\
& =\left(\frac{1}{n(n-1)} \sum_{\substack{u, u=1 \\
u \neq v}}^{n} \mathscr{L}_{u}^{p} \oplus \mathscr{L}_{u}^{q}\right)^{1 / p+q} .
\end{aligned}
$$

In 1982, Dombi [10] introduced a generator and produced Dombi T-norm and Dombi T-conorm shown as

$$
\begin{aligned}
& \check{\mathrm{T}}_{(D, \ell)}=\frac{1}{1+\left((1-\alpha / \alpha)^{k}+(\beta / 1-\beta)^{k}\right)^{1 / \kappa}}, \\
& \check{\mathrm{T}}_{(\mathscr{D}, \ell)}^{*}=\frac{1}{1+\left((\alpha / 1-\alpha)^{\kappa}+(1-\beta / \beta)^{k}\right)^{1 / k}},
\end{aligned}
$$

where $k>0, \alpha, \beta \in[0,1]$.

\section{Complex Intuitionistic Fuzzy Dombi Bonferroni Mean Operators}

In this section, we develop some new operators based on Bonferroni mean operators and Dombi operational laws to aggregate the CIFNs. Some desirable properties of the 
developed operators are discussed, and various cases are investigated in detail.

Definition 7. Let $\mathscr{L}_{u}=\left\langle\left\langle\Upsilon_{u}, \eta_{u}\right\rangle,\left\langle\Lambda_{u}, \lambda_{u}\right\rangle\right\rangle(u=1,2$, $\ldots, n)$ be CIFNs, $p, q \geq 0, k>0$. Then, a mapping $\mathrm{CIFDBM}^{p, q}: \mathscr{L}^{n} \longrightarrow \mathscr{L}$ is called a complex intuitionistic fuzzy Dombi Bonferroni mean operator if

$$
\begin{aligned}
& \operatorname{CIFDBM}^{p, q}\left(\mathscr{L}_{1}, \mathscr{L}_{2}, \ldots, \mathscr{L}_{n}\right) \\
& =\left(\frac{1}{n(n-1)} \sum_{\substack{u, v=1 \\
u \neq v}}^{n} \mathscr{L}_{u}^{p} \otimes_{\mathscr{D}} \mathscr{L}_{v}^{q}\right)^{1 / p+q} .
\end{aligned}
$$

Theorem 1. Let $\mathscr{L}_{u}=\left\langle\left\langle\Upsilon_{u}, \eta_{u}\right\rangle,\left\langle\Lambda_{u}, \lambda_{u}\right\rangle\right\rangle \quad(u=1,2$, $\ldots, n)$ be CIFNs, $p, q \geq 0, k>0$. Then, the resultant aggregated values by using the CIFDBM ${ }^{2, q}$ operator is a CIFN, and

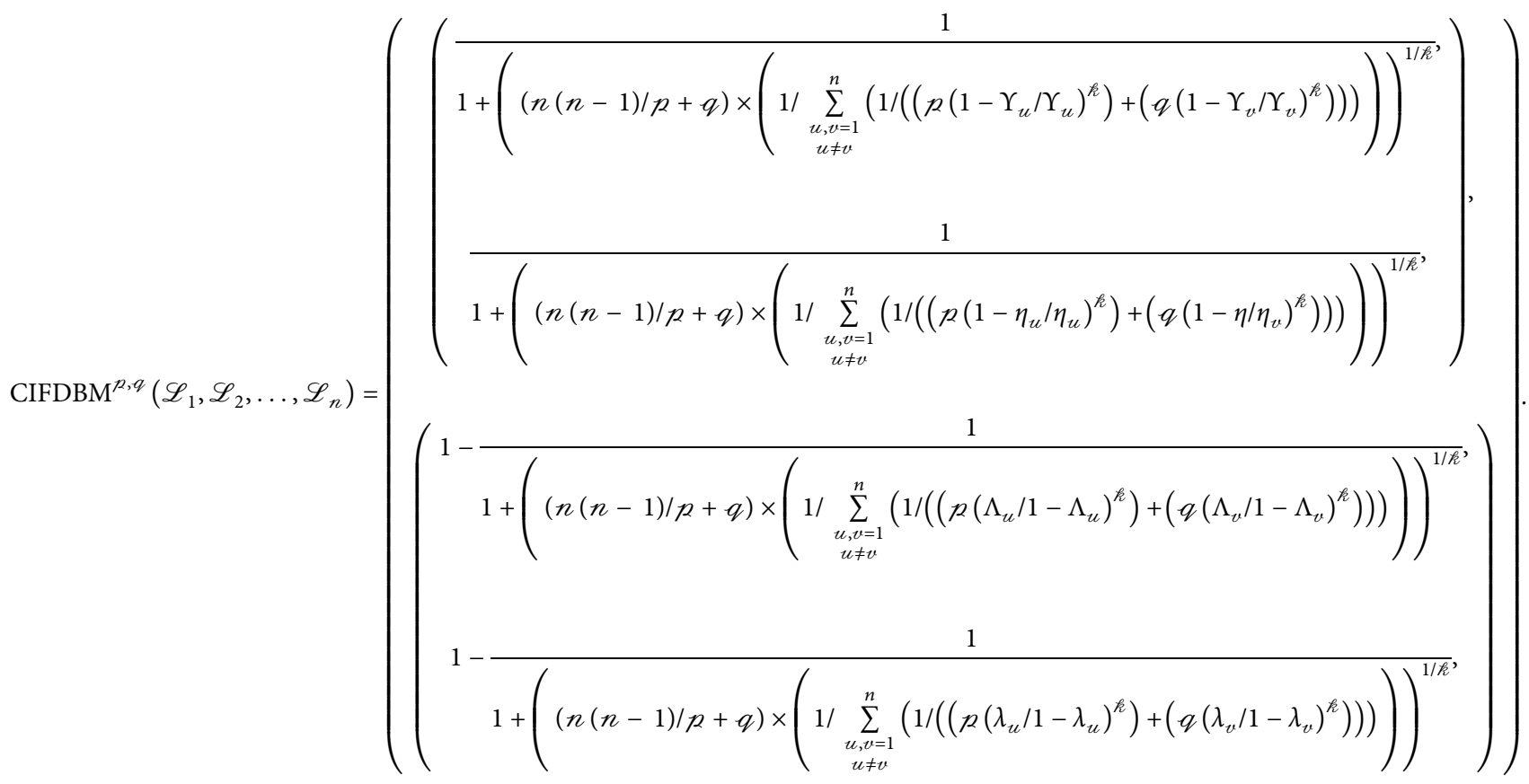

Proof. First, we prove that the above equation holds. Since

$$
\mathscr{L}_{u}^{R}=\left(\left(\frac{1}{1+\left(p\left(1-\Upsilon_{u} / \Upsilon_{u}\right)^{R}\right)^{1 / \hbar}}, \frac{1}{1+\left(p\left(1-\eta_{u} / \eta_{u}\right)^{R}\right)^{1 / \hbar}}\right),\left(1-\frac{1}{1+\left(p\left(\Lambda_{u} / 1-\Lambda_{u}\right)^{R}\right)^{1 / \hbar}}, 1-\frac{1}{1+\left(p\left(\lambda_{u} / 1-\lambda_{u}\right)^{R}\right)^{1 / \hbar}}\right)\right),
$$

and

$$
\mathscr{L}_{v}^{R}=\left(\left(\frac{1}{1+\left(q\left(1-\Upsilon_{v} / \Upsilon_{v}\right)^{/ /}\right)^{1 / \hbar}}, \frac{1}{1+\left(q\left(1-\eta_{v} / \eta_{v}\right)^{k}\right)^{1 / \hbar}}\right),\left(1-\frac{1}{1+\left(q\left(\Lambda_{v} / 1-\Lambda_{v}\right)^{k}\right)^{1 / \hbar}}, 1-\frac{1}{1+\left(q\left(\lambda_{v} / 1-\lambda_{v}\right)^{k}\right)^{1 / \hbar}}\right)\right),
$$


Now, let $\quad\left(1-\Upsilon_{u} / \Upsilon_{u}\right)=\mu_{u}, \quad\left(1-\Upsilon_{u} / \Upsilon_{v}\right)=\mu_{\vartheta}, \quad\left(\Lambda_{v} / 1-\Lambda_{v}\right)=\tau_{\vartheta},\left(\lambda_{u} / 1-\lambda_{u}\right)=\rho_{u}$, and $\left(\lambda_{v} / 1-\lambda_{v}\right)=\rho_{v}$. $\left(1-\eta_{u} / \eta_{u}\right)=v_{u}, \quad\left(1-\eta_{u} / \eta_{u}\right)=v_{u}, \quad\left(\Lambda_{u} / 1-\Lambda_{u}\right)=\tau_{u}, \quad$ Then,

$$
\mathscr{L}_{u}^{R}=\left(\left(\frac{1}{1+(p)^{1 / \hbar} \mu_{u}}, \frac{1}{1+(p)^{1 / \hbar} \nu_{u}}\right),\left(1-\frac{1}{1+(p)^{1 / \hbar} \tau_{u}}, 1-\frac{1}{1+(p)^{1 / \hbar} \rho_{u}}\right)\right)
$$

and

$$
\mathscr{L}_{u}^{p}=\left(\left(\frac{1}{1+(q)^{1 / \hbar} \mu_{v}}, \frac{1}{1+(q)^{1 / \hbar} v_{v}}\right),\left(1-\frac{1}{1+(q)^{1 / \hbar} \tau_{v}}, 1-\frac{1}{1+(q)^{1 / \hbar} \rho_{v}}\right)\right) .
$$

It implies that

$$
\mathscr{L}_{u}^{R} \otimes_{\mathscr{D}} \mathscr{L}_{u}^{R}=\left(\left(\frac{1}{1+\left(p \mu_{u}^{k}+q \mu_{u}^{k}\right)^{1 / \hbar}}, \frac{1}{1+\left(p v_{u}^{k}+q v_{u}^{k}\right)^{1 / \hbar}}\right),\left(1-\frac{1}{1+\left(p \tau_{u}^{k}+q \tau_{v}^{k}\right)^{1 / \hbar}}, 1-\frac{1}{1+\left(p \rho_{u}^{k}+q \rho_{u}^{k}\right)^{1 / \hbar}}\right)\right),
$$

$$
\begin{aligned}
& \sum_{\substack{u, v=1 \\
u \neq v}}^{n} \mathscr{L}_{u}^{R} \otimes_{\mathscr{D}} \mathscr{L}_{u}^{q}=\left(\left(1-\frac{1}{1+\left(\sum_{\substack{u, u=1 \\
u \neq v}}^{n}\left(1 /\left(p \mu_{u}^{k}+q \mu_{v}^{k}\right)\right)\right)^{1 / \hbar}}, 1-\frac{1}{1+\left(\sum_{\substack{u, v=1 \\
u \neq v}}^{n}\left(1 /\left(p v_{u}^{k}+q v_{v}^{k}\right)\right)\right)^{1 / \hbar}}\right),\right. \\
& \left(\frac{1}{1+\left(\sum_{\substack{u, v=1 \\
u \neq u}}^{n}\left(1 /\left(p \tau_{u}^{k}+q \tau_{u}^{k}\right)\right)\right)^{1 / \hbar}}, \frac{1}{1+\left(\sum_{\substack{u, u=1 \\
u \neq v}}^{n}\left(1 /\left(p \rho_{u}^{k}+q \rho_{u}^{k}\right)\right)\right)^{1 / \hbar}}\right) .
\end{aligned}
$$


Furthermore,

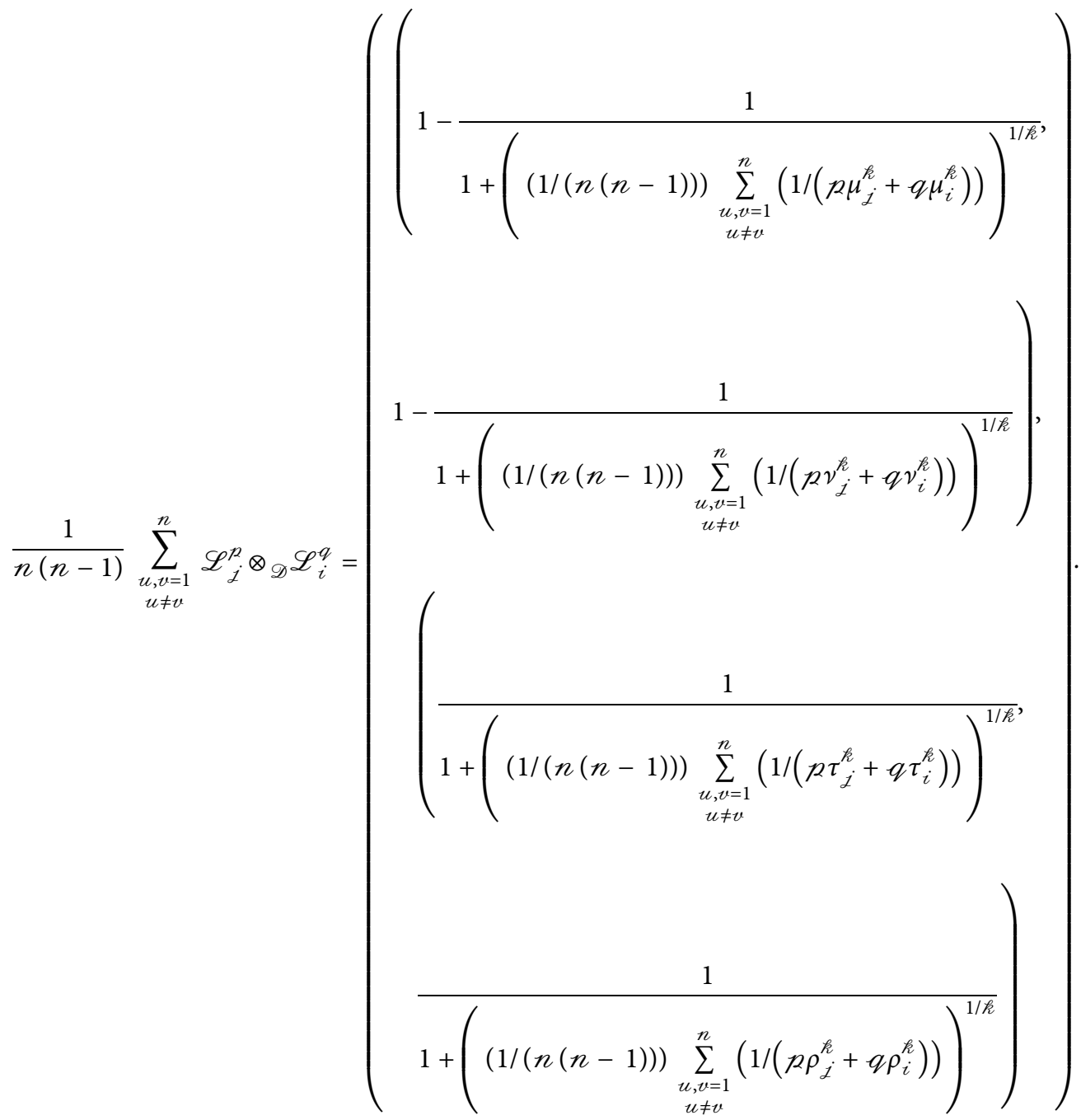


Then,

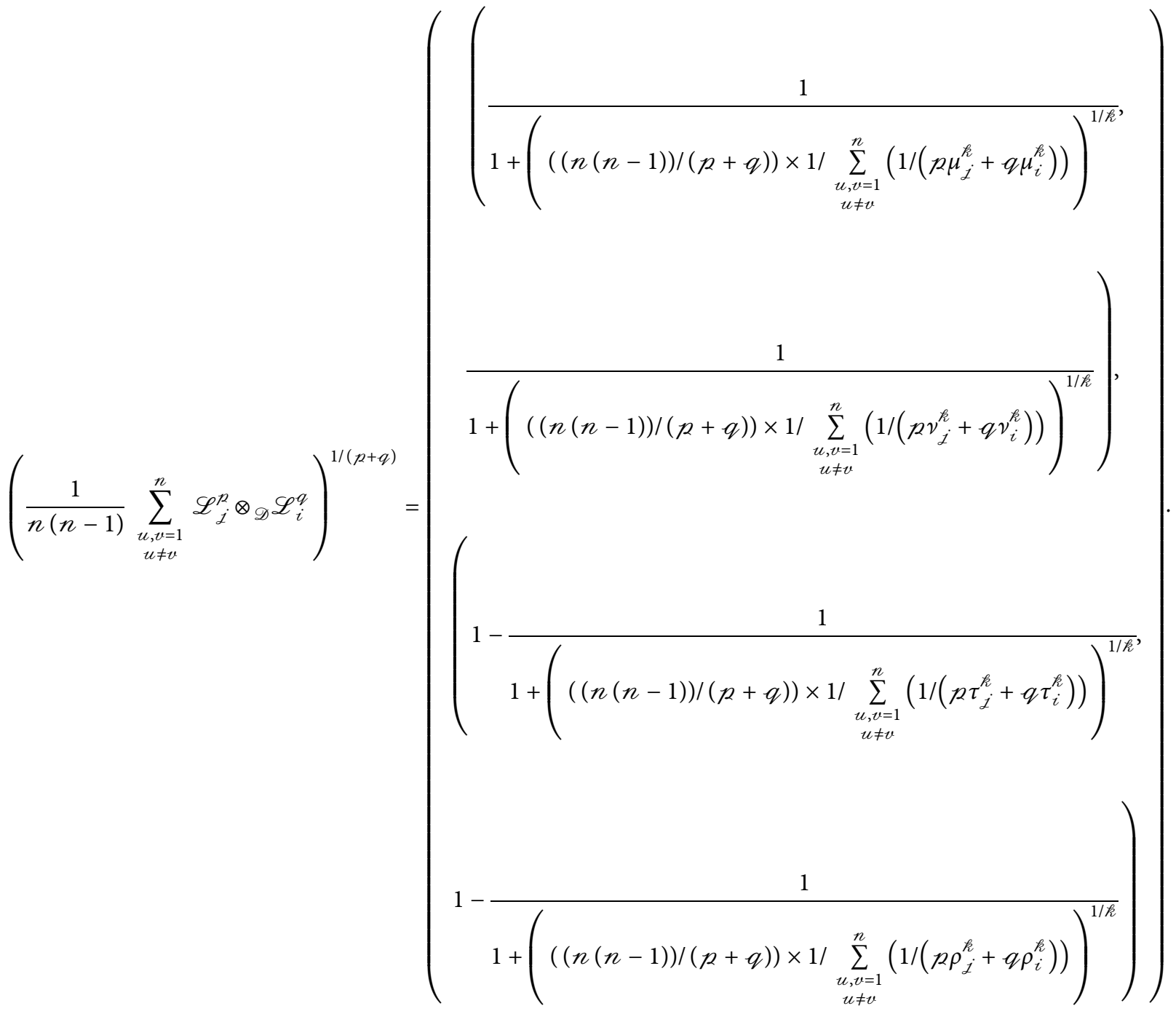

Put, $\quad\left(\left(1-\Upsilon_{j}\right) / \Upsilon_{j}\right)=\mu_{j}, \quad\left(\left(1-\Upsilon_{i}\right) / \Upsilon_{i}\right)=\mu_{i}, \quad \tau_{j}, \quad\left(\left(1-\Lambda_{i}\right) / \Lambda_{i}\right)=\tau_{i}, \quad\left(\lambda_{j} /\left(1-\lambda_{j}\right)\right)=\rho_{j}, \quad$ and $\left(\left(1-\eta_{j}\right) / \eta_{j}\right)=v_{j}, \quad\left(\left(1-\eta_{i}\right) / \eta_{i}\right)=v_{i}, \quad\left(\left(1-\Lambda_{j}\right) / \Lambda_{j}\right)=\quad\left(\lambda_{i} /\left(1-\lambda_{i}\right)\right)=\rho_{i}$. Then, 
$\left(\frac{1}{n(n-1)} \sum_{\substack{u, u=1 \\ u \neq u}}^{n} \mathscr{L}_{i}^{p} \otimes_{D^{2}} \mathscr{L}_{i}^{q}\right)^{1 /(p+q)}$

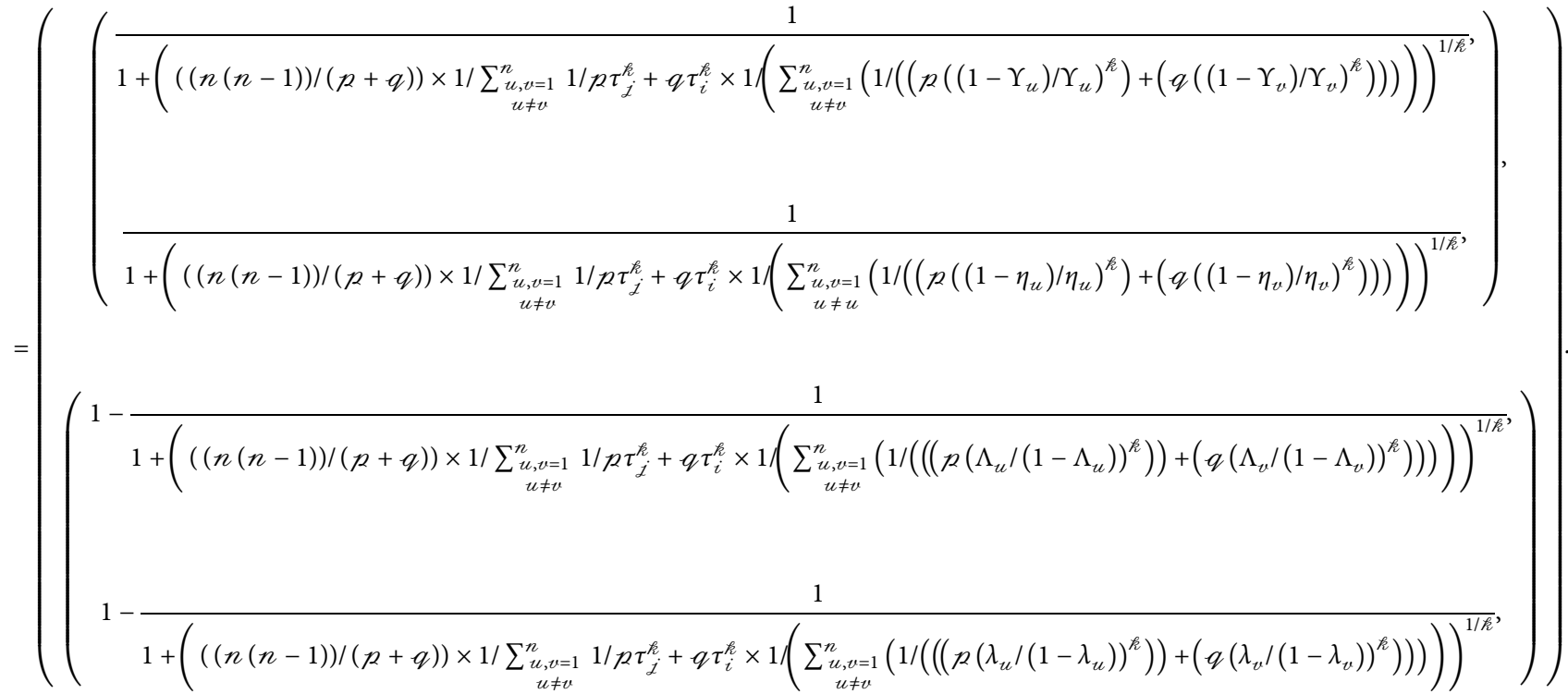

Next, we prove that equation (9) is CIFN. Let

$$
\begin{aligned}
& \alpha=\frac{1}{1+\left(((n(n-1)) /(p+q)) \times 1 /\left(\sum_{\substack{u, v=1 \\
u \neq v}}^{n}\left(1 /\left(\left(p\left(1-\Upsilon_{u} / \Upsilon_{u}\right)^{\kappa}\right)+\left(q\left(1-\Upsilon_{v} / \Upsilon_{v}\right)^{\kappa}\right)\right)\right)\right)\right)^{1 / \kappa}}, \\
& \beta=\frac{1}{1+\left(((n(n-1)) /(p+q)) \times 1 /\left(\sum_{\substack{u, v=1 \\
u \neq v}}^{n}\left(1 /\left(\left(p\left(1-\eta_{u} / \eta_{u}\right)^{k}\right)+\left(q\left(1-\eta_{u} / \eta_{u}\right)^{k}\right)\right)\right)\right)\right)^{1 / \hbar}}, \\
& \gamma=1-\frac{1}{1+\left(((n(n-1)) /(p+q)) \times 1\left(\sum_{\substack{u, u=1 \\
u \neq v}}^{n}\left(1 /\left(\left(p\left(\Lambda_{u} / 1-\Lambda_{u}\right)^{k}\right)+\left(q\left(\Lambda_{u} / 1-\Lambda_{u}\right)^{k}\right)\right)\right)\right)\right)^{1 / \hbar}},
\end{aligned}
$$

and 


$$
\delta=1-\frac{1}{1+\left(((n(n-1)) /(p+q)) \times 1 /\left(\sum_{\substack{u, u=1 \\ u \neq v}}^{n}\left(1 /\left(\left(p\left(\lambda_{u} / 1-\lambda_{u}\right)^{\kappa}\right)+\left(q\left(\lambda_{v} / 1-\lambda_{v}\right)^{\kappa}\right)\right)\right)\right)\right)^{1 / k}} .
$$

Next, we prove that CVMD and CVNMD fulfill the condition as follows:

(a): $\alpha, \beta, \gamma, \delta \in[0,1](b): 0 \leq \alpha+\gamma \leq 1$ and $0 \leq \beta+\delta \leq 1$. (a) First, we prove part (a). Since $\mu_{j}, \mu_{i}, \tau_{j}$, and $\tau_{i}$ are monotonically decreasing functions, $v_{j}, v_{i}, \rho_{j}$, and $\rho_{i}$ are monotonically increasing functions, and $k>0$.

$$
\mu_{j}, \mu_{i}, \tau_{j}, \tau_{i}>0 \Rightarrow\left(\sum_{\substack{u, u=1 \\ u \neq v}}^{n} \frac{1}{\left(p \mu_{j}^{k}+q \mu_{i}^{k}\right)}\right)^{1 / \kappa}>0 \text { and }\left(\sum_{\substack{u, u=1 \\ u \neq v}}^{n} \frac{1}{\left(p \tau_{j}^{k}+q \tau_{i}^{k}\right)}\right)^{1 / \hbar}>0 .
$$

As

$$
\begin{aligned}
\frac{n(n-1)}{p+q} \geq 0 & \Rightarrow\left(\frac{n(n-1)}{p+q}\right)^{1 / \hbar} \\
& \times \frac{1}{\left(\sum_{\substack{u, v=1 \\
u \neq v}}^{n}\left(1 /\left(p \mu_{j}^{k}+q \mu_{i}^{k}\right)\right)\right)^{1 / \hbar}} \geq 0,
\end{aligned}
$$

and

$$
\left(\frac{n(n-1)}{p+q}\right)^{1 / \kappa} \times \frac{1}{\left(\sum_{\substack{u, v=1 \\ u \neq v}}^{n}\left(1 /\left(p \tau_{j}^{k}+q \tau_{i}^{\kappa}\right)\right)\right)^{1 / \hbar}} \geq 0
$$

Then, we have

$$
1+\left(\frac{n(n-1)}{p+q}\right)^{1 / \hbar} \times \frac{1}{\left(\sum_{\substack{u, u=1 \\ u \neq v}}^{n}\left(1 /\left(p \mu_{j}^{k}+q \mu_{i}^{k}\right)\right)\right)^{1 / \hbar}} \geq 1,
$$

and

$$
1+\left(\frac{n(n-1)}{p+q}\right)^{1 / \hbar} \times \frac{1}{\left(\sum_{\substack{u, v=1 \\ u \neq v}}^{n}\left(1 /\left(p \tau_{j}^{k}+q \tau_{i}^{k}\right)\right)\right)^{1 / k}} \geq 1 .
$$

It implies that

$$
\begin{aligned}
& 0 \leq \frac{1}{1+((n(n-1)) /(p+q))^{1 / \hbar} \times\left(1 /\left(\sum_{\substack{u, v=1 \\
u \neq v}}^{n}\left(1 /\left(p \mu_{\dot{j}}^{k}+q \mu_{i}^{k}\right)\right)\right)^{1 / \hbar}\right)} \leq 1, \\
& 0 \leq \frac{1}{((n(n-1)) /(p+q))^{1 / \hbar} \times\left(1 /\left(\sum_{\substack{u, v=1 \\
u \neq v}}^{n}\left(1 /\left(p \tau_{j}^{k}+q \tau_{i}^{k}\right)\right)\right)^{1 / \hbar}\right)} \leq 1 .
\end{aligned}
$$


That is $0 \leq \alpha \leq 1$, and $0 \leq \gamma \leq 1$. In the similar way, we can show that $0 \leq \beta \leq 1$, and $0 \leq \delta \leq 1$.

(b) We prove that $0 \leq \alpha+\gamma \leq 1$ and $0 \leq \beta+\delta \leq 1$.

Since $0 \leq \alpha \leq 1,0 \leq \gamma \leq 1$ and $0 \leq \beta \leq 1,0 \leq \delta \leq 1$. We need only to prove that $0 \leq \alpha+\gamma \leq 1$ and $0 \leq \beta+\delta \leq 1$ for this we have, as $0 \leq \mu_{j}+\tau_{j}, \mu_{i}+\tau_{i} \leq 1$, and $0 \leq \nu_{j}+\rho_{j}, v_{i}+\rho_{i} \leq 1$, then, $1-\tau_{j} \geq \mu_{j}, 1-\tau_{i} \geq \mu_{i}$, and $1-\rho_{j} \geq v_{j}, 1-\rho_{i} \geq v_{i}$ implies that $\left(1-\mu_{j} / \mu_{j}\right) \geq \tau_{j} / 1-\tau_{j},\left(1-\mu_{i} / \mu_{i}\right) \geq \tau_{i} / 1-\tau_{i}$, $\left(1-\rho_{j} / \rho_{j}\right) \geq v_{j} / 1-v_{j}$, and $\left(1-\rho_{i} / \rho_{i}\right) \geq v_{i} / 1-v_{i}$. That is, $\alpha_{j} \geq \gamma_{j}, \alpha_{i} \geq \gamma_{i}, \beta_{j} \geq \delta_{j}$, and $\beta_{i} \geq \delta_{i}$. Furthermore, we can get

$$
\left(\sum_{\substack{u, v=1 \\ u \neq v}}^{n} \frac{1}{\left(p \mu_{j}^{k}+q \mu_{i}^{k}\right)}\right)^{1 / \hbar} \leq\left(\sum_{\substack{u, v=1 \\ u \neq v}}^{n} \frac{1}{\left(p \tau_{\dot{j}}^{k}+q \tau_{i}^{k}\right)}\right)^{1 / \hbar},
$$

and

$$
\begin{aligned}
& \left(\sum_{\substack{u, v=1 \\
u \neq v}}^{n} \frac{1}{\left(p v_{j}^{k}+q v_{i}^{k}\right)}\right)^{1 / \kappa} \leq\left(\sum_{\substack{u, v=1 \\
u \neq v}}^{n} \frac{1}{\left(p \rho_{j}^{k}+q \rho_{i}^{k}\right)}\right)^{1 / \kappa} \\
& 1+\left(\frac{n(n-1)}{p+q}\right)^{1 / \hbar} \times \frac{1}{\left(\sum_{\substack{u, v=1 \\
u \neq v}}^{n}\left(1 /\left(p \mu_{j}^{k}+q \mu_{i}^{k}\right)\right)\right)^{1 / \hbar}} \geq 1+\left(\frac{n(n-1)}{p+q}\right)^{1 / \hbar} \times \frac{1}{\left(\sum_{\substack{u, v=1 \\
u \neq v}}^{n}\left(1 /\left(p \tau_{j}^{k}+q \tau_{i}^{k}\right)\right)\right)^{1 / \hbar}}, \\
& 1+\left(\frac{n(n-1)}{p+q}\right)^{1 / \hbar} \times \frac{1}{\left(\sum_{\substack{u, u=1 \\
u \neq v}}^{n}\left(1 /\left(p v_{j}^{\hbar}+q v_{i}^{\kappa}\right)\right)\right)^{1 / \hbar}} \geq 1+\left(\frac{n(n-1)}{p+q}\right)^{1 / \hbar} \times \frac{1}{\left(\sum_{\substack{u, v=1 \\
u \neq v}}^{n}\left(1 /\left(p \rho_{j}^{k}+q \rho_{i}^{\kappa}\right)\right)\right)^{1 / \hbar}}
\end{aligned}
$$

and

$$
\begin{gathered}
\left.\frac{1}{1+((n(n-1)) /(p+q))^{1 / \hbar} \times\left(1 /\left(\sum_{\substack{u, v=1 \\
u \neq v}}^{n}\left(1 /\left(p \mu_{j}^{k}+q \mu_{i}^{k}\right)\right)\right)^{1 / \hbar}\right)}\right) \\
\left.\leq \frac{1}{1+((n(n-1)) /(p+q))^{1 / \hbar} \times\left(1 /\left(\sum_{\substack{u, v=1 \\
u \neq v}}^{n}\left(1 /\left(p \tau_{j}^{k}+q \tau_{i}^{k}\right)\right)\right)^{1 / \hbar}\right)}\right)
\end{gathered}
$$




$$
\begin{aligned}
& \frac{1}{1+((n(n-1)) /(p+q))^{1 / \hbar} \times\left(1 /\left(\sum_{\substack{u, u=1 \\
u \neq v}}^{n}\left(1 /\left(p v_{j}^{k}+q v_{i}^{k}\right)\right)\right)^{1 / \hbar}\right)} \\
& \leq \frac{1}{1+((n(n-1)) /(p+q))^{1 / \hbar} \times\left(1 /\left(\sum_{\substack{u, v=1 \\
u \neq v}}^{n}\left(1 /\left(p \rho_{j}^{k}+q \rho_{i}^{k}\right)\right)\right)^{1 / \hbar}\right)} \text {. }
\end{aligned}
$$

So,

$$
\begin{aligned}
& 0 \leq \frac{1}{1+((n(n-1)) /(p+q))^{1 / \hbar} \times\left(1 /\left(\sum_{\substack{u, v=1 \\
u \neq v}}^{n}\left(1 /\left(p \mu_{j}^{\hbar}+q \mu_{i}^{k}\right)\right)\right)^{1 / \hbar}\right)}+1 \\
& -\frac{1}{1+((n(n-1)) /(p+q))^{1 / \hbar} \times\left(1 /\left(\sum_{\substack{u, u=1 \\
u \neq v}}^{n}\left(1 /\left(p \tau_{j}^{k}+q \tau_{i}^{k}\right)\right)\right)^{1 / \hbar}\right)} \leq 1,
\end{aligned}
$$

and

$$
\begin{aligned}
& 0 \leq \frac{1}{1+((n(n-1)) /(p+q))^{1 / \hbar} \times\left(1 /\left(\sum_{\substack{u, u=1 \\
u \neq v}}^{n}\left(1 /\left(p v_{j}^{k}+q v_{i}^{k}\right)\right)\right)^{1 / \hbar}\right)}+1 \\
& -\frac{1}{1+((n(n-1)) /(p+q))^{1 / \hbar} \times\left(1 /\left(\sum_{\substack{u, v=1 \\
u \neq v}}^{n}\left(1 /\left(p \rho_{j}^{k}+q \rho_{i}^{k}\right)\right)\right)^{1 / \hbar}\right)} \leq 1 .
\end{aligned}
$$

Thus, $1 \geq \alpha+\gamma \geq 0$ and $1 \geq \beta+\delta \geq 0$. Hence from (a) and (b), we conclude that equation (9), is a CIFN. For
CIFDBM $^{p, q}$, some cases regarding parameters $p$ and $q$ are presented as follows: 
Case $a$. When $p \longrightarrow 0, k>0$, then we can have

$\operatorname{CIFDBM}^{0, q}\left(\mathscr{L}_{1}, \mathscr{L}_{2}, \ldots, \mathscr{L}_{n}\right)$

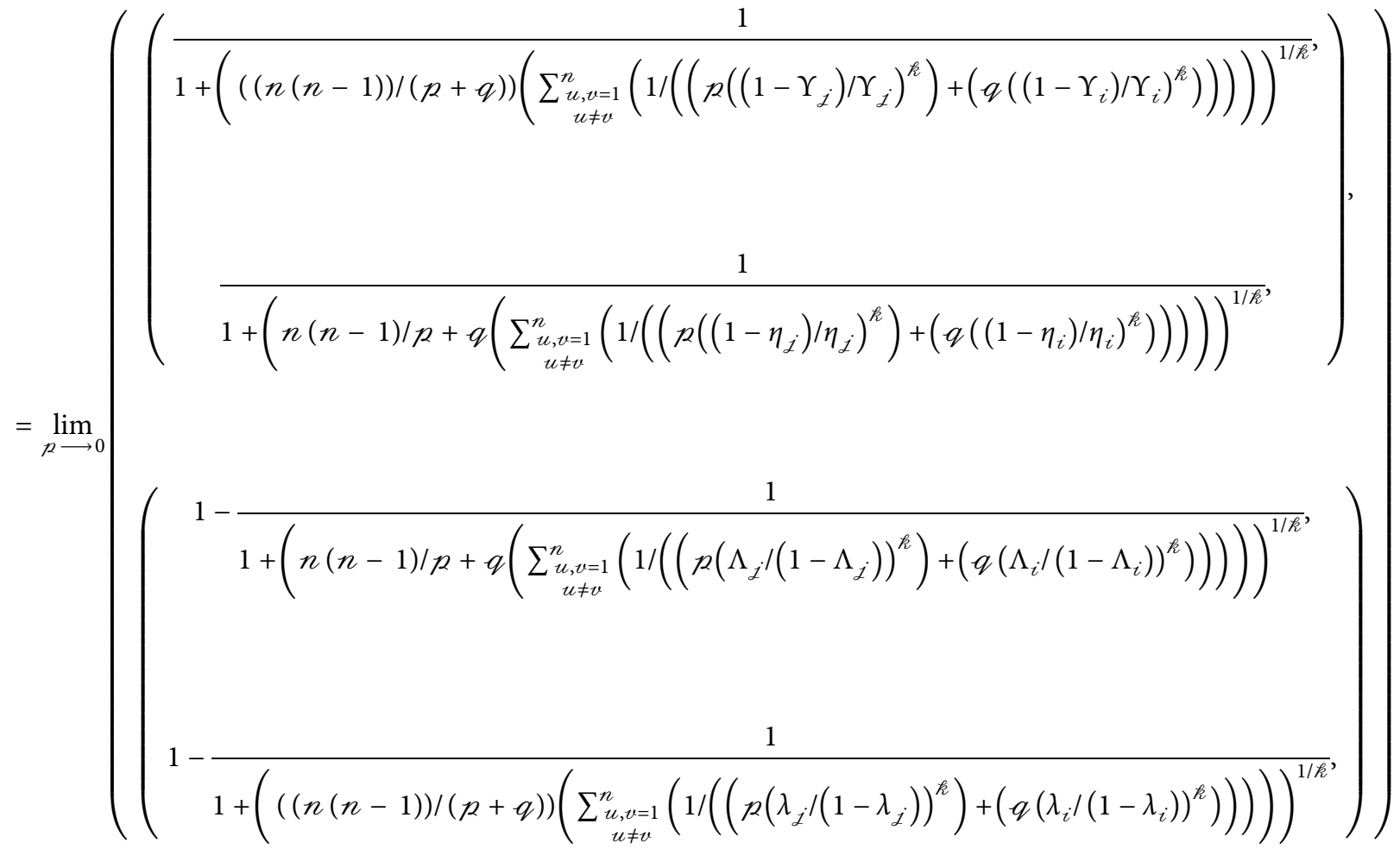

$=\left(\begin{array}{c}\frac{1}{1+\left(((n(n-1)) /(p+q))\left(\sum_{\substack{u, u=1 \\ u \neq u}}^{n}\left(1 /\left(q\left(\left(1-\Upsilon_{i}\right) / \Upsilon_{i}\right)^{/}\right)\right)\right)\right)^{1 / \hbar}}, \\ \frac{1}{1+\left(((n(n-1)) /(p+q))\left(\sum_{\substack{u, u=1 \\ u \neq u}}^{n}\left(1 /\left(q\left(\left(1-\eta_{i}\right) / \eta_{i}\right)^{/}\right)\right)\right)\right)^{1 / \hbar}},\end{array}\right)$,

$$
\left.\left(\begin{array}{c}
1-\frac{1}{1+\left(((n(n-1)) /(p+q))\left(\sum_{\substack{u, v=1 \\
u \neq v}}^{n}\left(1 /\left(q\left(\Lambda_{i} /\left(1-\Lambda_{i}\right)\right)^{/}\right)\right)\right)\right)^{1 / \hbar}}, \\
1-\frac{1}{1+\left(((n(n-1)) /(p+q))\left(\sum_{\substack{u, u=1 \\
u \neq v}}^{n}\left(1 /\left(q\left(\lambda_{i} /\left(1-\lambda_{i}\right)\right)^{/}\right)\right)\right)\right)^{1 / \hbar}}
\end{array}\right)\right)
$$


Case $b$. When $p=1, q \longrightarrow 0, k>0$, then we can have

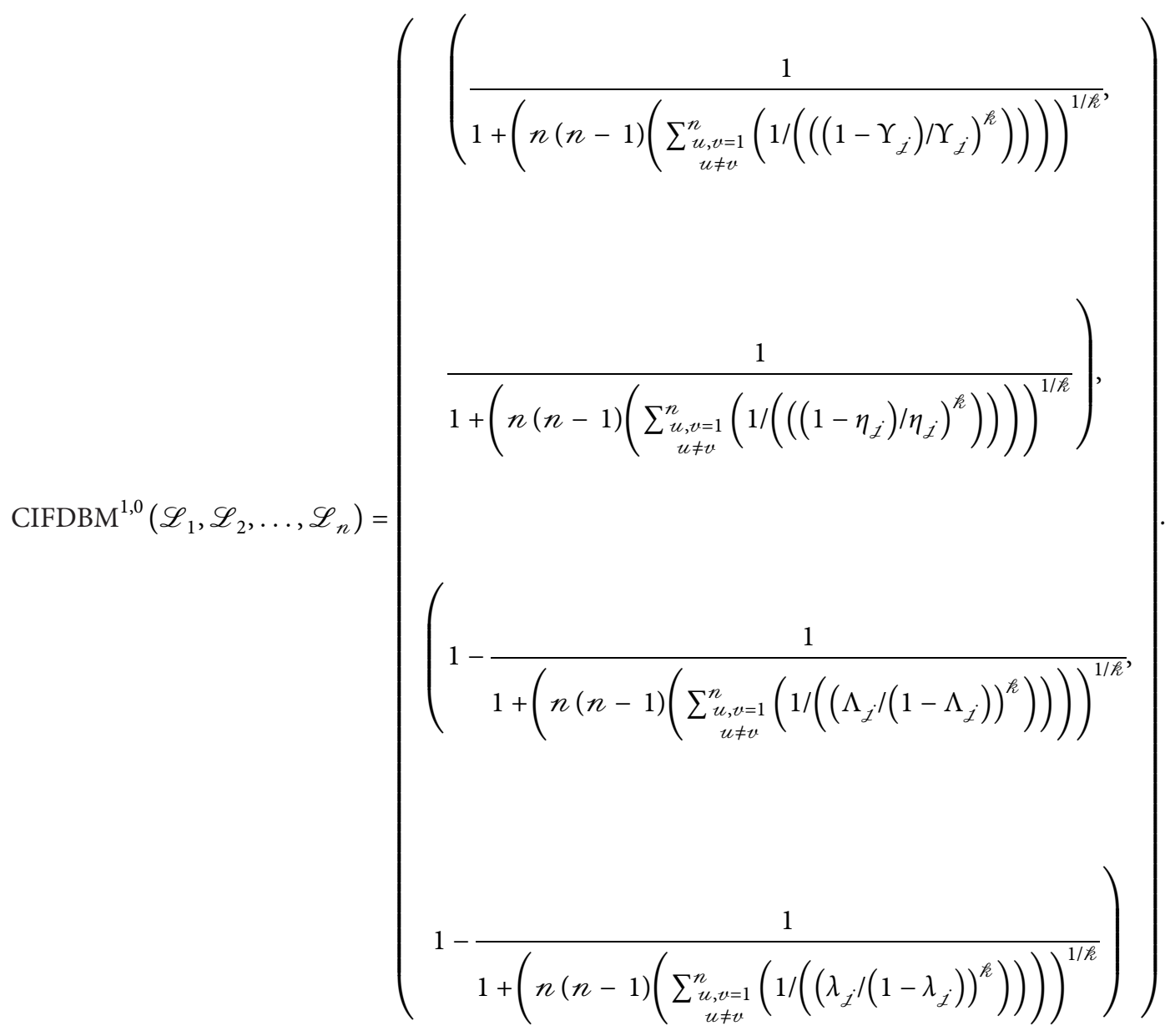


Case c. When $q \longrightarrow 0, k>0$, then we can have

$\operatorname{CIFDBM}^{p, 0}\left(\mathscr{L}_{1}, \mathscr{L}_{2}, \ldots, \mathscr{L}_{n}\right)$

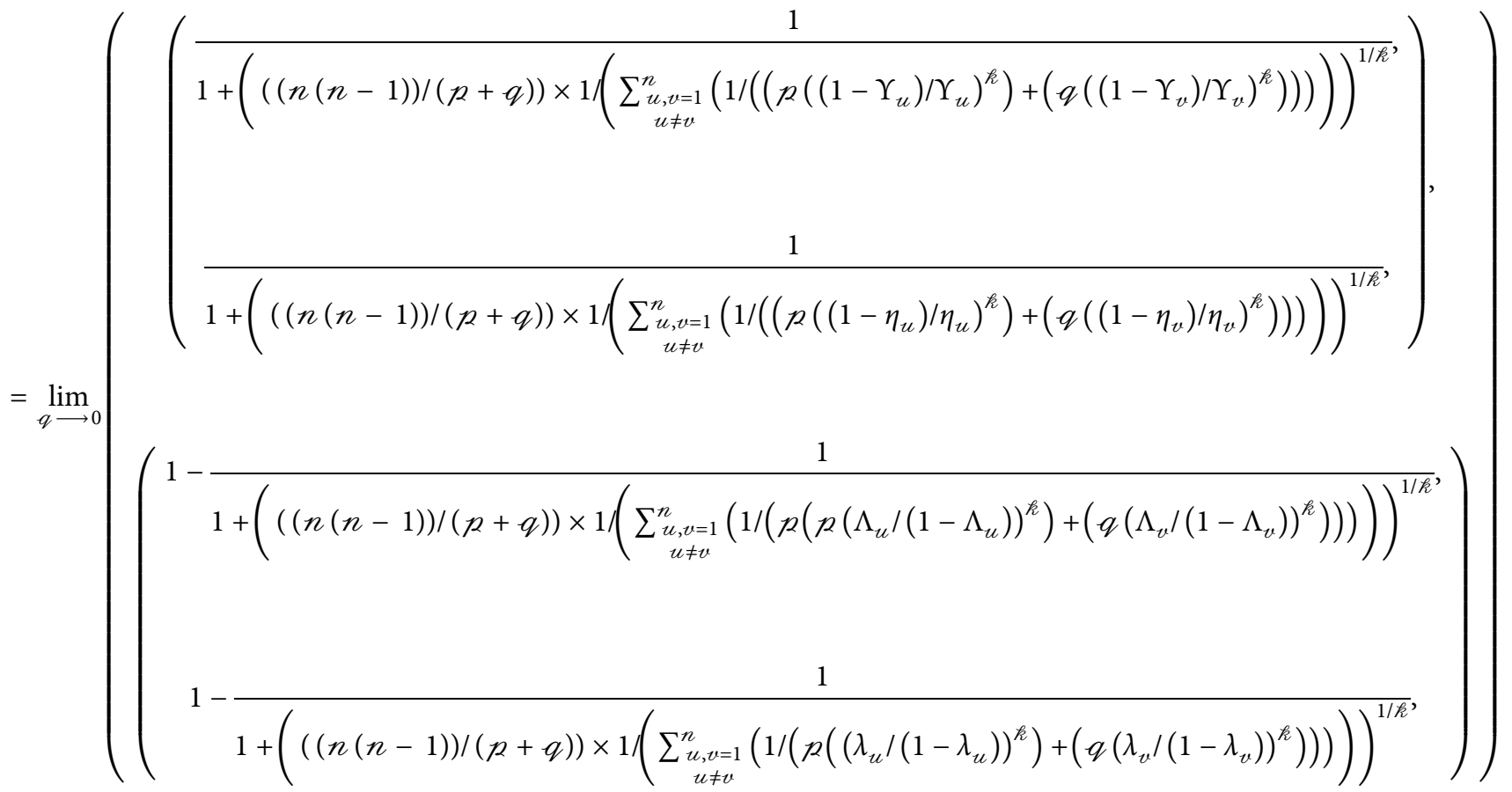

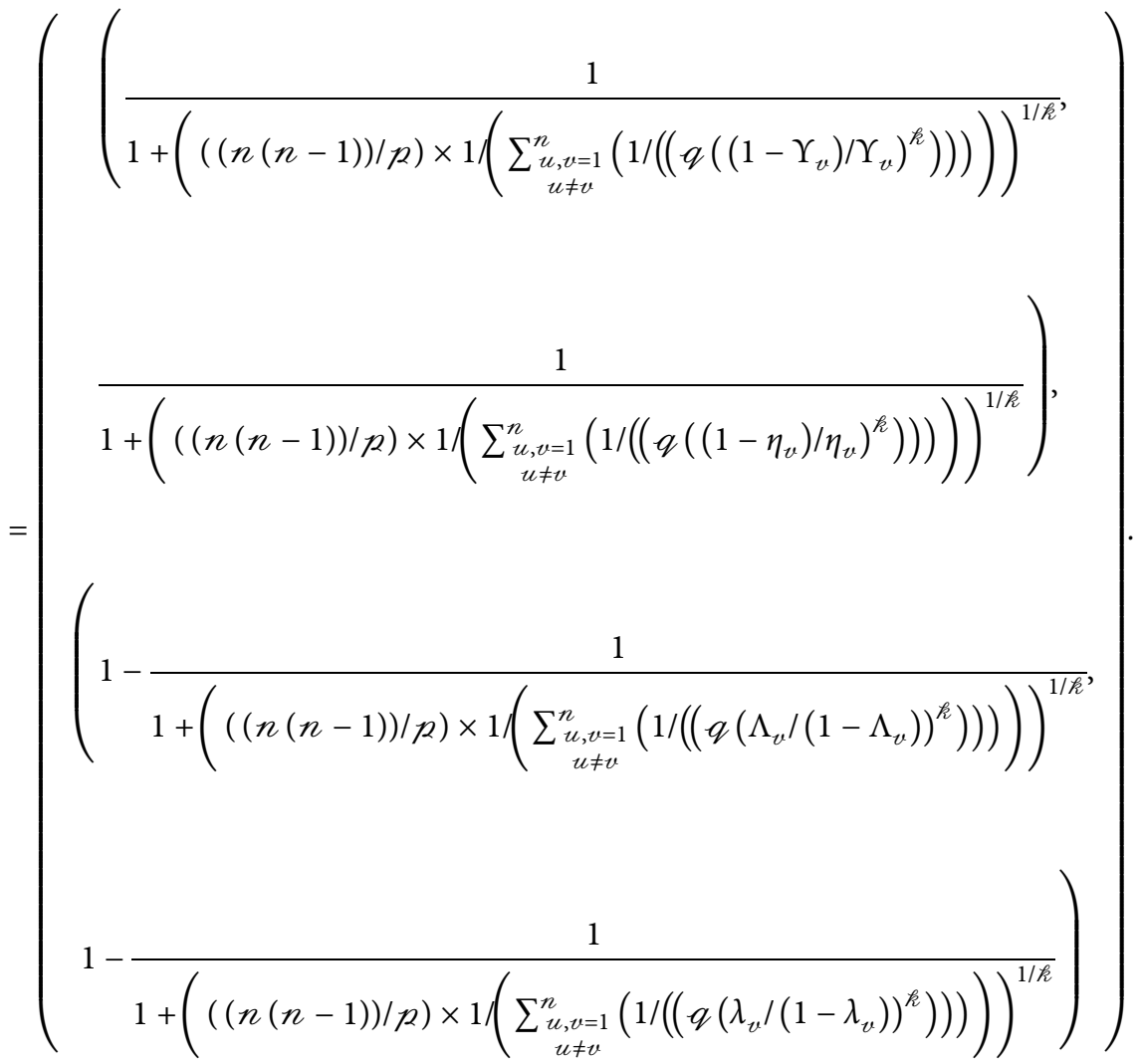


Case $d$. When $p=q=1, k>0$, then we can get

$$
\begin{aligned}
& \operatorname{CIFDBM}^{1,1}\left(\mathscr{L}_{1}, \mathscr{L}_{2}, \ldots, \mathscr{L}_{n}\right)
\end{aligned}
$$

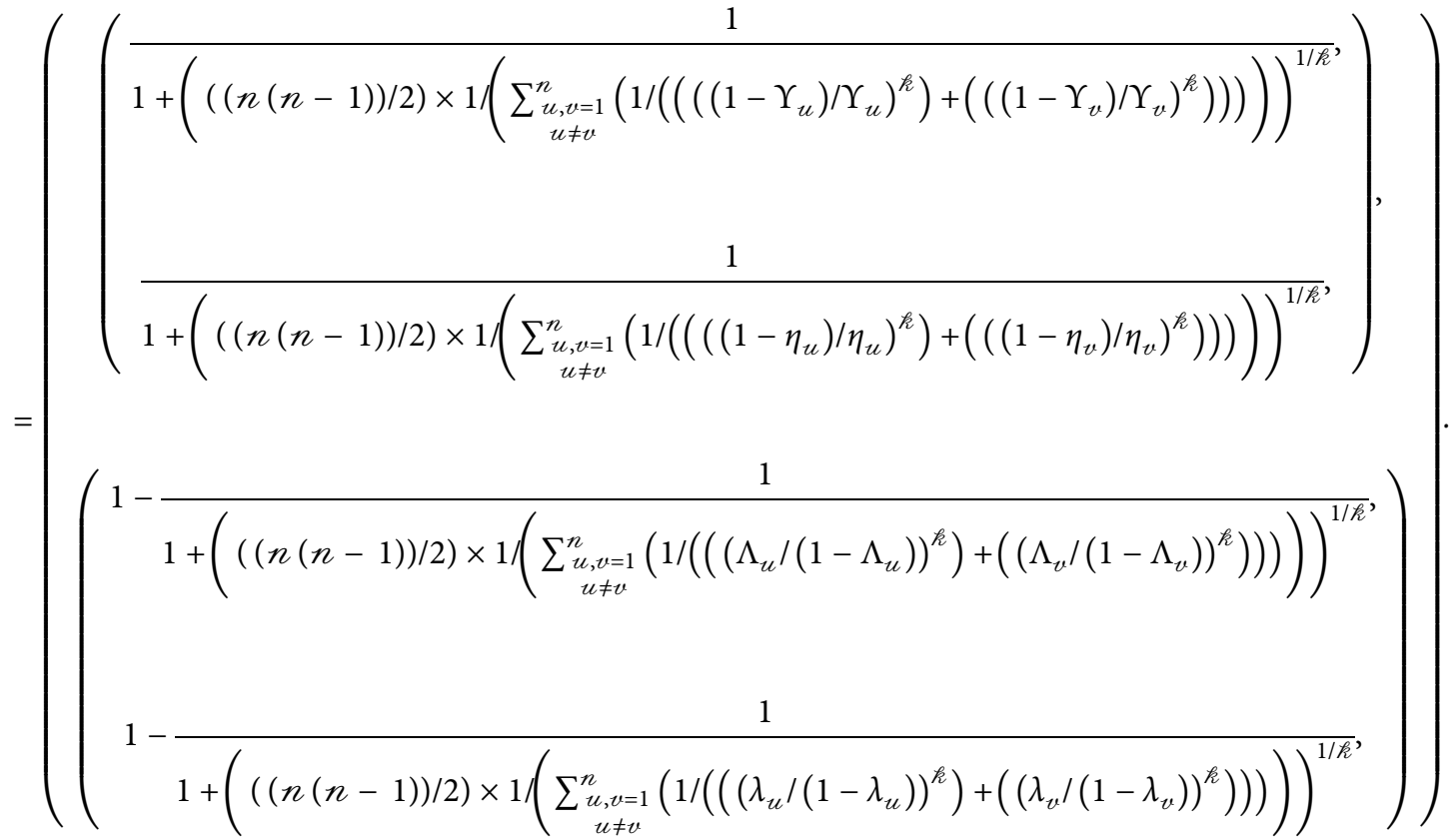

Example 1. Let $\mathscr{L}_{1}=\left\langle 0.3 e^{i 2 \pi 0.4}, 0.5 e^{i 2 \pi 0.6}\right\rangle, \mathscr{L}_{2}=\left\langle 0.5 e^{i 2 \pi 0.5}\right.$, $\left.0.3 e^{i 2 \pi 0.5}\right\rangle$, and $\mathscr{L}_{3}=\left\langle 0.3 e^{i 2 \pi 0.3}, 0.2 e^{i 2 \pi 0.2}\right\rangle$ be three CIFNS. Then, we use the CIFDBM to aggregate the three CIFNs and generate a comprehensive value. The steps are shown as follows: (suppose $p=1, q=1=2$, and $k=2$ ). Since

$$
\begin{aligned}
\sum_{\substack{u, u=1 \\
u \neq v}}^{3} \frac{1}{\left(p\left(\left(1-\Upsilon_{u}\right) / \Upsilon_{u}\right)^{k}\right)+\left(q\left(\left(1-\Upsilon_{u}\right) / \Upsilon_{u}\right)^{k}\right)}= & \frac{1}{\left(1 \times\left(\left(1-\Upsilon_{1}\right) / \Upsilon_{1}\right)^{2}\right)+\left(1 \times\left(\left(1-\Upsilon_{2}\right) / \Upsilon_{2}\right)^{2}\right)} \\
& +\frac{1}{\left(1 \times\left(\left(1-\Upsilon_{1}\right) / \Upsilon_{1}\right)^{2}\right)+\left(1 \times\left(\left(1-\Upsilon_{3}\right) / \Upsilon_{3}\right)^{2}\right)} \\
& +\frac{1}{\left(1 \times\left(\left(1-\Upsilon_{2}\right) / \Upsilon_{2}\right)^{2}\right)+\left(1 \times\left(\left(1-\Upsilon_{1}\right) / \Upsilon_{1}\right)^{2}\right)} \\
& +\frac{1}{\left(1 \times\left(\left(1-\Upsilon_{2}\right) / \Upsilon_{2}\right)^{2}\right)+\left(1 \times\left(\left(1-\Upsilon_{3}\right) / \Upsilon_{3}\right)^{2}\right)} \\
& +\frac{1}{\left(1 \times\left(\left(1-\Upsilon_{3}\right) / \Upsilon_{3}\right)^{2}\right)+\left(1 \times\left(\left(1-\Upsilon_{1}\right) / \Upsilon_{1}\right)^{2}\right)} \\
& +\frac{1}{\left(1 \times\left(\left(1-\Upsilon_{3}\right) / \Upsilon_{3}\right)^{2}\right)+\left(1 \times\left(\left(1-\Upsilon_{2}\right) / \Upsilon_{2}\right)^{2}\right)}=0.8044
\end{aligned}
$$




$$
\begin{aligned}
& \frac{n(n-1)}{p+q} \times 1 /\left(\sum_{\substack{u, v=1 \\
u \neq v}}^{n} \frac{1}{\left(p\left(\left(1-\Upsilon_{u}\right) / \Upsilon_{u}\right)^{k}\right)+\left(q\left(\left(1-\Upsilon_{v}\right) / \Upsilon_{v}\right)^{k}\right)}\right) \\
& \quad=\frac{3(3-1)}{1+1} \times \frac{1}{\left(\sum_{\substack{u, v=1 \\
u \neq v}}^{3}\left(1 /\left(\left(1 \times\left(\left(1-\Upsilon_{u}\right) / \Upsilon_{u}\right)^{2}\right)+\left(1 \times\left(\left(1-\Upsilon_{, v}\right) / \Upsilon_{, v}\right)^{2}\right)\right)\right)\right)}=1.9312,
\end{aligned}
$$

1

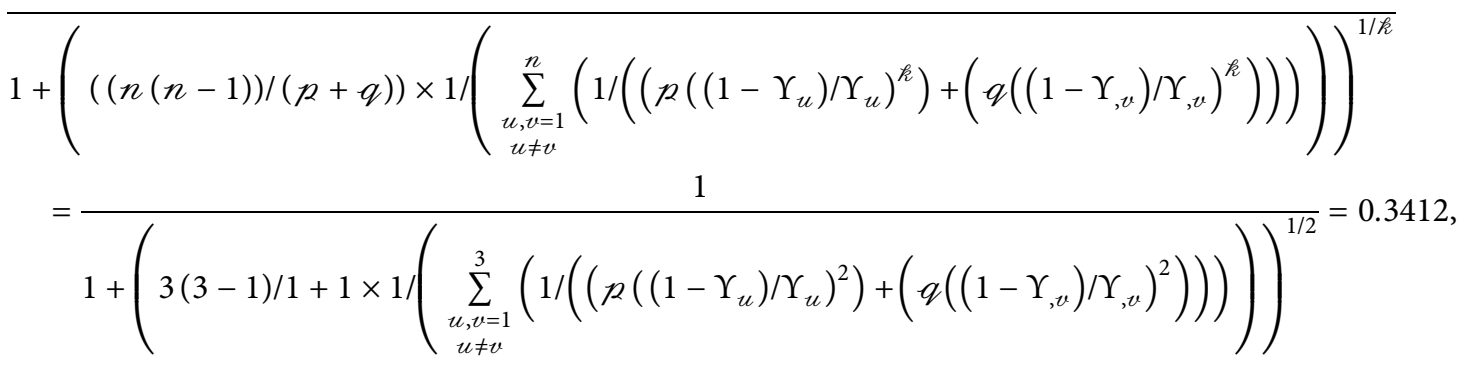

1

$1+\left(((n(n-1)) /(p+q)) \times 1 /\left(\sum_{\substack{u, v=1 \\ u \neq v}}^{n}\left(1 /\left(\left(p\left(\left(1-\eta_{u}\right) / \eta_{u}\right)^{k}\right)+\left(q\left(\left(1-\eta_{i}\right) / \eta_{i}\right)^{k}\right)\right)\right)\right)\right)^{1 / k}$
$=\frac{1}{1+\left(((3(3-1)) /(1+1)) \times 1 /\left(\sum_{\substack{u, v=1 \\ u \neq v}}^{3}\left(1 /\left(\left(1 \times\left(\left(1-\eta_{u}\right) / \eta_{u}\right)^{2}\right)+\left(1 \times\left(\left(1-\eta_{u}\right) / \eta_{u}\right)^{1}\right)\right)\right)\right)\right)^{1 / 2}}=0.3860$,

$$
\begin{aligned}
& 1-\frac{1}{1+\left(((n(n-1)) /(p+q)) \times 1 /\left(\sum_{\substack{u, u=1 \\
u \neq v}}^{n}\left(1 /\left(\left(p\left(\Lambda_{u} /\left(1-\Lambda_{u}\right)\right)^{\kappa}\right)+\left(q\left(\Lambda_{v} /\left(1-\Lambda_{v}\right)\right)^{\kappa}\right)\right)\right)\right)\right)^{1 / \kappa}} \\
& =1-\frac{1}{1+\left(3(3-1) / 1+1 \times 1 /\left(\sum_{\substack{u, u=1 \\
u \neq v}}^{3}\left(1 /\left(\left(1 \times\left(\Lambda_{u} /\left(1-\Lambda_{u}\right)\right)^{\kappa}\right)+\left(1 \times\left(\Lambda_{u} /\left(1-\Lambda_{v}\right)\right)^{2}\right)\right)\right)\right)\right)^{1 / 2}}=0.3362 \text {, }
\end{aligned}
$$

$$
\begin{aligned}
& 1-\frac{1}{1+\left(((n(n-1)) /(p+q)) \times 1 /\left(\sum_{\substack{u, u=1 \\
u \neq v}}^{n}\left(1 /\left(\left(p\left(\lambda_{u} /\left(1-\lambda_{u}\right)\right)^{k}\right)+\left(q\left(\lambda_{u} /\left(1-\lambda_{v}\right)\right)^{k}\right)\right)\right)\right)\right)^{1 / \kappa}} \\
& =1-\frac{1}{1+\left(((3(3-1)) /(1+1)) \times 1 /\left(\sum_{\substack{u, v=1 \\
u \neq v}}^{3}\left(1 /\left(\left(1 \times\left(\lambda_{u} /\left(1-\lambda_{u}\right)\right)^{2}\right)+\left(1 \times\left(\lambda_{u} /\left(1-\lambda_{v}\right)\right)^{2}\right)\right)\right)\right)\right)^{1 / 2}}=0.4857 .
\end{aligned}
$$

We present some desirable properties for the $\mathrm{CIFDBM}^{p, q}$ operator as follows.

Idempotency 1 . Let $\mathscr{L}_{u}=\left\langle\left\langle\Upsilon_{u}, \eta_{u}\right\rangle,\left\langle\Lambda_{u}, \lambda_{u}\right\rangle\right\rangle$ $(u=1,2, \ldots, n)$ be CIFNs and $p, q \geq 0, k>0$. If
$\mathscr{L}_{1}=\mathscr{L}_{2}=\ldots=\mathscr{L}_{n}$, that is, $\mathscr{L}_{u}=\mathscr{L}=\langle\langle\Upsilon, \eta\rangle,\langle\Lambda, \lambda\rangle\rangle$, then

$$
\operatorname{CIFDBM}^{p, q}\left(\mathscr{L}_{1}, \mathscr{L}_{2}, \ldots, \mathscr{L}_{n}\right)=\mathscr{L}
$$


Proof. Suppose

$\operatorname{CIFDBM}^{p, q}\left(\mathscr{L}_{1}, \mathscr{L}_{2}, \ldots, \mathscr{L}_{n}\right)=\langle\langle\alpha, \beta\rangle,\langle\gamma, \delta\rangle\rangle$. First, we prove that $\alpha=\Upsilon, \beta=\eta$. Since $\Upsilon_{u}=\Upsilon, \eta_{u}=\eta$ and $\Upsilon_{u}=\Upsilon$, $\eta_{u}=\eta$, therefore,

$$
\begin{aligned}
& \alpha=\frac{1}{1+\left(((n(n-1)) /(p+q)) \times\left(1 /\left(\sum_{\substack{u, v=1 \\
u \neq v}}^{n}\left(1 /\left(\left(p(((1-\Upsilon) / \Upsilon))^{\kappa}\right)+\left(q(((1-\Upsilon) / \Upsilon))^{\AA}\right)\right)\right)\right)\right)\right)^{1 / \kappa}} \\
& 1 \\
& =\frac{1+\left(((n(n-1)) /(p+q)) \times\left(1 /\left(\sum_{\substack{u, u=1 \\
u \neq v}}^{n}\left(1 /\left(\left(p(((1-\Upsilon) / \Upsilon))^{k}\right)+\left(q(((1-\Upsilon) / \Upsilon))^{k}\right)\right)\right)\right)\right)\right)^{1 / k}}{} \\
& =\frac{1}{1+\left(((n(n-1)) /(p+q)) \times\left(1 /\left(\sum_{\substack{u, v=1 \\
u \neq v}}^{n}\left(1 /\left(\left((((1-\Upsilon) / \Upsilon))^{k}\right)+(p+q)\right)\right)\right)\right)\right.}=\frac{1}{1+((1-\Upsilon) / \Upsilon)}=\Upsilon \text {, } \\
& \beta=\frac{1}{1+\left(((n(n-1)) /(p+q)) \times\left(1 /\left(\sum_{\substack{u, u=1 \\
u \neq v}}^{n}\left(1 /\left(\left(p\left(\left(1-\eta_{u}\right) / \eta_{u}\right)^{k}\right)+\left(q\left(\left(1-\eta_{v}\right) / \eta_{v}\right)^{k}\right)\right)\right)\right)\right)\right)^{1 / \hbar}} \\
& =\frac{1}{1+\left(((n(n-1)) /(p+q)) \times\left(1 /\left(\sum_{\substack{u, v=1 \\
u \neq v}}^{n}\left(1 /\left(\left(p((1-\eta) / \eta)^{\kappa}\right)+\left(q((1-\eta) / \eta)^{k}\right)\right)\right)\right)\right)\right)^{1 / \hbar}}
\end{aligned}
$$

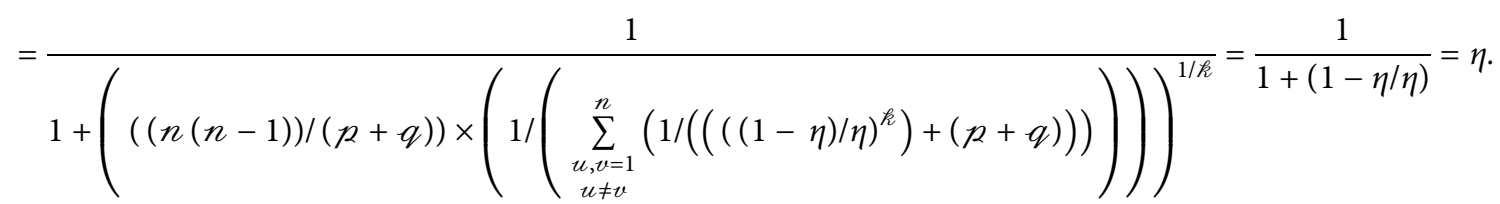

This means that $(\alpha, \beta)=(\Upsilon, \eta)$.

In the similar way, we can also prove that $(\gamma, \delta)=(\Lambda, \lambda)$. This shows that

$$
\operatorname{CIFDBM}^{p, q}\left(\mathscr{L}_{1}, \mathscr{L}_{2}, \ldots, \mathscr{L}_{n}\right)=\langle\langle\alpha, \beta\rangle,\langle\gamma, \delta\rangle\rangle=\langle\langle\Upsilon, \eta\rangle,\langle\Lambda, \lambda\rangle\rangle=\mathscr{L} .
$$

Monotonicity 1 . Let $\mathscr{L}_{u}=\left\langle\left\langle\Upsilon_{u}, \eta_{u}\right\rangle,\left\langle\Lambda_{u}, \lambda_{u}\right\rangle\right\rangle$ and $\mathscr{L}_{u}^{\prime}=\left\langle\left\langle\Upsilon_{u}^{\prime}, \eta_{u}^{\prime}\right\rangle,\left\langle\Lambda_{u}^{\prime}, \lambda_{u}^{\prime}\right\rangle\right\rangle$ and $(u=1,2, \ldots, n)$ be two collections of CIFNs, $p, q \geq 0, k>0$. If $\Upsilon_{u} \geq \Upsilon_{u}^{\prime}, \eta_{u} \geq \eta_{u}^{\prime}$, $\Lambda_{u} \geq \Lambda_{u}^{\prime}$, and $\lambda_{u} \geq \lambda_{u}^{\prime}$ for all $\left.u=1,2, \ldots, n\right)$, then $\operatorname{CIFDBM}^{p, q}\left(\mathscr{L}_{1}, \mathscr{L}_{2}, \ldots, \mathscr{L}_{n}\right) \geq \operatorname{CIFDBM}^{p, q}\left(\mathscr{L}_{1}^{\prime}, \mathscr{L}_{2}^{\prime}, \ldots, \mathscr{L}_{n}^{\prime}\right)$.
Proof. Let CIFDBM ${ }^{p, q}\left(\mathscr{L}_{1}, \mathscr{L}_{2}, \ldots, \mathscr{L}_{n}\right)=\langle\langle\Upsilon, \eta\rangle,\langle\Lambda, \lambda\rangle\rangle$ and $\operatorname{CIFDBM}^{p, q}\left(\mathscr{L}_{1}^{\prime}, \mathscr{L}_{2}^{\prime}, \ldots, \mathscr{L}_{n}^{\prime}\right)=\left\langle\left\langle Y^{\prime}, \quad \eta^{\prime}\right\rangle,\left\langle\Lambda^{\prime}, \lambda^{\prime}\right\rangle\right\rangle$. We need to prove that $\Upsilon \geq \Upsilon^{\prime}, \eta \geq \eta^{\prime}, \Lambda \geq \Lambda^{\prime}$, and $\lambda \geq \lambda^{\prime}$. Since $\Upsilon_{u} \geq \Upsilon_{u}^{\prime}, \eta_{u} \geq \eta_{u}^{\prime}, \Lambda_{u} \geq \Lambda_{u}^{\prime}$, and $\lambda_{u} \geq \lambda_{u}^{\prime}$ for all $u=1,2, \ldots, n$, then we have $\left(\left(1-\Upsilon_{u}\right) / \Upsilon_{u}\right) \leq \quad\left(\left(1-\Upsilon_{u}^{\prime}\right) / \Upsilon_{u}^{\prime}\right)$, $\left(\left(1-\eta_{u}\right) / \eta_{u}\right) \leq\left(\left(1-\eta_{u}^{\prime}\right) / \eta_{u}^{\prime}\right) \quad$ and $\quad\left(\left(1-\Upsilon_{u}\right) / \Upsilon_{i}\right) \leq$ $\left(\left(1-\Upsilon_{i}^{\prime}\right) / \Upsilon_{v}^{\prime}\right),\left(\left(1-\eta_{v}\right) / \eta_{v}\right) \leq\left(\left(1-\eta_{v}^{\prime}\right) / \eta_{v}^{\prime}\right)$. So, we have 


$$
\begin{aligned}
& \left(\left(\sum_{\substack{u, u=1 \\
u \neq v}}^{n}\left(1 /\left(\left(p\left(\left(1-\Upsilon_{u}\right) / \Upsilon_{u}\right)^{k}\right)+\left(q\left(\left(1-\Upsilon_{u}\right) / \Upsilon_{v}\right)^{k}\right)\right)\right)\right)\right)^{1 / \hbar} \\
& \leq\left(\left(\sum_{\substack{u, v=1 \\
u \neq v}}^{n}\left(1 /\left(\left(p\left(\left(1-\Upsilon_{u}^{\prime}\right) / \Upsilon_{u}^{\prime}\right)^{\kappa}\right)+\left(q\left(\left(1-\Upsilon_{u}^{\prime}\right) / \Upsilon_{u}^{\prime}\right)^{k}\right)\right)\right)\right)\right)^{1 / \hbar},
\end{aligned}
$$

and

$$
\begin{aligned}
& \left(\left(\sum_{\substack{u, u=1 \\
u \neq u}}^{n}\left(1 /\left(\left(p\left(\left(1-\eta_{u}\right) / \eta_{u}\right)^{k}\right)+\left(q\left(\left(1-\eta_{u}\right) / \eta_{u}\right)^{k}\right)\right)\right)\right)\right)^{1 / \hbar} \\
& \leq\left(\left(\sum_{\substack{u, v=1 \\
u \neq v}}^{n}\left(1 /\left(\left(p\left(\left(1-\eta_{u}^{\prime}\right) / \eta_{u}^{\prime}\right)^{R}\right)+\left(q\left(\left(1-\eta_{v}^{\prime}\right) / \eta_{v}^{\prime}\right)^{R}\right)\right)\right)\right)\right)^{1 / k}, \\
& 1+\left(((n(n-1)) /(p+q)) \times 1 /\left(\sum_{\substack{u, u=1 \\
u \neq u}}^{n}\left(1 /\left(\left(p\left(\left(1-\Upsilon_{u}\right) / \Upsilon_{u}\right)^{k}\right)+\left(q\left(\left(1-\Upsilon_{u}\right) / \Upsilon_{u}\right)^{k}\right)\right)\right)\right)\right)^{1 / \hbar} \\
& \leq 1+\left(((n(n-1)) /(p+q)) \times 1 /\left(\sum_{\substack{u, u=1 \\
u \neq v}}^{n}\left(1 /\left(\left(p\left(\left(1-\Upsilon_{u}^{\prime}\right) / \Upsilon_{u}^{\prime}\right)^{\kappa}\right)+\left(q\left(\left(1-\Upsilon_{u}^{\prime}\right) / \Upsilon_{u}^{\prime}\right)^{\star}\right)\right)\right)\right)\right)^{1 / \hbar},
\end{aligned}
$$

and

$$
\begin{aligned}
& 1+\left(((n(n-1)) /(p+q)) \times 1 /\left(\sum_{\substack{u, v=1 \\
u \neq v}}^{n}\left(1 /\left(\left(p\left(\left(1-\eta_{u}\right) / \eta_{u}\right)^{\kappa}\right)+\left(q\left(\left(1-\eta_{u}\right) / \eta_{u}\right)^{k}\right)\right)\right)\right)\right)^{1 / \kappa} \\
& \leq 1+\left(((n(n-1)) /(p+q)) \times 1 /\left(\sum_{\substack{u, v=1 \\
u \neq v}}^{n}\left(1 /\left(\left(p\left(\left(1-\eta_{u}^{\prime}\right) / \eta_{u}^{\prime}\right)^{/}\right)+\left(q\left(\left(1-\eta_{u}^{\prime}\right) / \eta_{u}^{\prime}\right)^{/}\right)\right)\right)\right)\right)^{1 / \kappa} .
\end{aligned}
$$


Thus,

1

$$
\begin{aligned}
& 1+\left(((n(n-1)) /(p+q)) \times 1 /\left(\sum_{\substack{u, v=1 \\
u \neq v}}^{n}\left(1 /\left(\left(p\left(\left(1-\Upsilon_{u}\right) / \Upsilon_{u}\right)^{k}\right)+\left(q\left(\left(1-\Upsilon_{u}\right) / \Upsilon_{u}\right)^{k}\right)\right)\right)\right)\right)^{1 / \hbar} \\
& \geq \frac{1}{1+\left(((n(n-1)) /(p+q)) \times 1 /\left(\sum_{\substack{u, u=1 \\
u \neq v}}^{n}\left(1 /\left(\left(p\left(\left(1-\Upsilon_{u}^{\prime}\right) / \Upsilon_{u}^{\prime}\right)^{k}\right)+\left(q\left(\left(1-\Upsilon_{u}^{\prime}\right) / \Upsilon_{u}^{\prime}\right)^{k}\right)\right)\right)\right)\right)^{1 / \hbar},}
\end{aligned}
$$

and

1

$$
\begin{aligned}
& 1+\left(((n(n-1)) /(p+q)) \times 1 /\left(\sum_{\substack{u, u=1 \\
u \neq v}}^{n}\left(1 /\left(\left(p\left(\left(1-\eta_{u}\right) / \eta_{u}\right)^{k}\right)+\left(q\left(\left(1-\eta_{v}\right) / \eta_{u}\right)^{k}\right)\right)\right)\right)\right)^{1 / \hbar} \\
& \geq \frac{1}{1+\left(((n(n-1)) /(p+q)) \times 1 /\left(\sum_{\substack{u, v=1 \\
u \neq v}}^{n}\left(1 /\left(\left(p\left(\left(1-\eta_{u}^{\prime}\right) / \eta_{u}^{\prime}\right)^{k}\right)+\left(q\left(\left(1-\eta_{v}^{\prime}\right) / \eta_{u}^{\prime}\right)^{k}\right)\right)\right)\right)\right)^{1 / \kappa}} .
\end{aligned}
$$

This shows that $\Upsilon \geq \Upsilon^{\prime}$ and $\eta \geq \eta$. In the similar way, we can show that $\Lambda_{u} \geq \Lambda_{u}^{\prime}$ and $\lambda_{u} \geq \lambda_{u}^{\prime}$. Therefore, we have $\operatorname{CIFDBM}^{p, q}\left(\mathscr{L}_{1}, \mathscr{L}_{2}, \ldots, \mathscr{L}_{n}\right) \geq \operatorname{CIFDBM}^{p, q}\left(\mathscr{L}_{1}^{\prime}, \mathscr{L}_{2}^{\prime}, \ldots, \mathscr{L}_{n}^{\prime}\right)$,

Boundedness 1. Let $\mathscr{L}_{u}=\left\langle\left\langle\Upsilon_{u}, \eta_{u}\right\rangle,\left\langle\Lambda_{u}, \lambda_{u}\right\rangle\right\rangle$

$$
\mathscr{L}^{-} \leq \operatorname{CIFDBM}^{p, q}\left(\mathscr{L}_{1}, \mathscr{L}_{2}, \ldots, \mathscr{L}_{n}\right) \leq \mathscr{L}^{+}
$$

Proof. Based on Property 1, we have

$$
\begin{aligned}
\mathscr{L}^{-}= & \operatorname{CIFDBM}^{p, q}\left(\mathscr{L}_{1}, \mathscr{L}_{2}, \ldots, \mathscr{L}_{n}\right) \text { and } \operatorname{CIFDBM}^{p, q} \\
& \left(\mathscr{L}_{1}, \mathscr{L}_{2}, \ldots, \mathscr{L}_{n}\right)=\mathscr{L}^{+} .
\end{aligned}
$$
$(u=1,2, \ldots, n)$ be CIFNsand $p, q \geq 0, k>0$ and $\mathscr{L}^{+}=$ $\left\langle\left\langle\max _{u} \Upsilon_{u}, \max _{u} \eta_{u}\right\rangle, \quad\left\langle\min _{u} \Lambda_{u}, \min _{u} \lambda_{u}\right\rangle\right\rangle, \mathscr{L}^{-}=$ $\left\langle\left\langle\min _{u} \Upsilon_{u}, \min _{u} \eta_{u}\right\rangle,\left\langle\max _{u} \Lambda_{u}, \max _{u} \lambda_{u}\right\rangle\right\rangle$. Then,

Also, by Property 2, we have

$$
\operatorname{CIFDBM}^{p, q}\left(\mathscr{L}_{1}^{+}, \mathscr{L}_{2}^{+}, \ldots, \mathscr{L}_{n}^{+}\right) \geq \operatorname{CIFDBM}^{p, q}\left(\mathscr{L}_{1}, \mathscr{L}_{2}, \ldots, \mathscr{L}_{n}\right) \geq \operatorname{CIFDBM}^{p, q}\left(\mathscr{L}_{1}^{-}, \mathscr{L}_{2}^{-}, \ldots, \mathscr{L}_{n}^{-}\right)
$$

Therefore, we can get $\mathscr{L}^{-} \leq \operatorname{CIFDBM}^{p, q}\left(\mathscr{L}_{1}, \mathscr{L}_{2}\right.$, $\left.\ldots, \mathscr{L}_{n}\right) \leq \mathscr{L}^{+}$.
Definition 8. Let $\mathscr{L}_{u}=\left\langle\left\langle\Upsilon_{u}, \eta_{u}\right\rangle,\left\langle\Lambda_{u}, \lambda_{u}\right\rangle\right\rangle(u=1,2$, $\ldots, n)$ be CIFNs and $p, q \geq 0, k>0$. Then, a mapping CIFWDBM $^{p, q}: \mathscr{L}^{n} \longrightarrow \mathscr{L}$ is called CIF weighted Dombi Bonferroni mean operator if

$$
\operatorname{CIFDBM}^{p, q}\left(\mathscr{L}_{1}, \mathscr{L}_{2}, \ldots, \mathscr{L}_{n}\right)=\left(\frac{1}{n(n-1)} \sum_{\substack{u, v=1 \\ u \neq v}}^{n}\left(\mathscr{E}_{u} \mathscr{L}_{u}\right)^{p} \otimes_{\mathscr{D}}\left(\mathscr{E}_{v} \mathscr{L}_{v}\right)^{q}\right)^{1 /(p+q)},
$$


where $\mathscr{E}=\left(\mathscr{E}_{1}, \mathscr{E}_{2} \ldots, \mathscr{E}_{n}\right)^{T}$ is a weighted vector of $\mathscr{L}_{u}=\left\langle\left\langle\Upsilon_{u}, \eta_{u}\right\rangle,\left\langle\Lambda_{u}, \lambda_{u}\right\rangle\right\rangle(u=1,2, \ldots, n)$ such that $1 \geq \mathscr{E}_{n} \geq 0, \sum_{u=1}^{n} \mathscr{E}_{u}=1$.

Theorem 2. Let $\mathscr{L}_{u}=\left\langle\left\langle\Upsilon_{u}, \eta_{u}\right\rangle,\left\langle\Lambda_{u}, \lambda_{u}\right\rangle\right\rangle \quad(u=1,2$, $\ldots, n)$ CIFNs and $p, q \geq 0, k>0$. Then, by using
CIFWDBM $M^{p, q}$ operators, the aggregated value is a CIFN and where $\mathscr{E}=\left(\mathscr{E}_{1}, \mathscr{E}_{2} \ldots, \mathscr{E}_{n}\right)^{T}$ is a weighted vector of $\mathscr{L}_{u}=$ $\left\langle\left\langle\Upsilon_{u}, \eta_{u}\right\rangle,\left\langle\Lambda_{u}, \lambda_{u}\right\rangle\right\rangle(u=1,2, \ldots, n)$ such that $1 \geq \mathscr{E}_{n} \geq$ $0, \sum_{u=1}^{n} \mathscr{E}_{u}=1$.

$\operatorname{CIFWDBM}^{\mathrm{p}, \mathrm{q}}\left(\mathscr{L}_{1}, \mathscr{L}_{2}, \ldots, \mathscr{L}_{n}\right)$

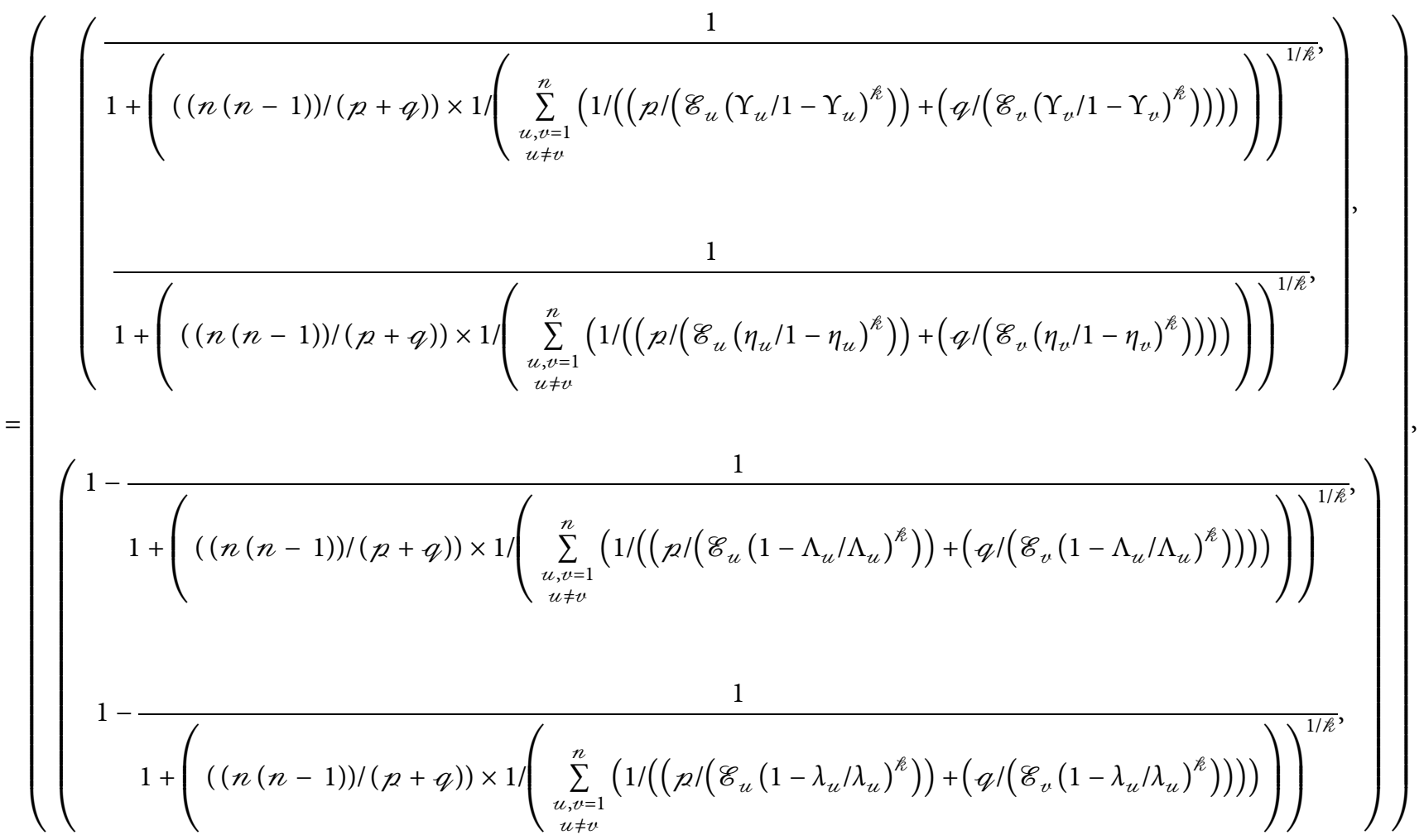

where $\mathscr{E}=\left(\mathscr{E}_{1}, \mathscr{E}_{2} \ldots, \mathscr{E}_{n}\right)^{T}$ is a weighted vector of $\mathscr{L}_{u}=$ $\left\langle\left\langle\Upsilon_{u}, \eta_{u}\right\rangle,\left\langle\Lambda_{u}, \lambda_{u}\right\rangle\right\rangle(u=1,2, \ldots, n) \quad$ such that $1 \geq \mathscr{E}_{n} \geq 0, \sum_{u=1}^{n} \mathscr{E}_{u}=1$.

Idempotency 2: let $\mathscr{L}_{u}=\left\langle\left\langle\Upsilon_{u}, \eta_{u}\right\rangle,\left\langle\Lambda_{u}, \lambda_{u}\right\rangle\right\rangle$ $(u=1,2, \ldots, n)$ be CIFNs and $p, q \geq 0, k>0$. If $\mathscr{L}_{1}=\mathscr{L}_{2}=\ldots=\mathscr{L}_{n}$, that is, $\mathscr{L}_{j}=\mathscr{L}=\langle\langle\Upsilon, \eta\rangle$, $\langle\Lambda, \lambda\rangle\rangle$, then

$$
\operatorname{CIFWDBM}^{p, q}\left(\mathscr{L}_{1}, \mathscr{L}_{2}, \ldots, \mathscr{L}_{n}\right)=\mathscr{L}
$$

Monotonicity 2: let $\mathscr{L}_{u_{1}}=\left\langle\left\langle\Upsilon_{u}, \eta_{u}\right\rangle,\left\langle\Lambda_{u}, \lambda_{u}\right\rangle\right\rangle$ $(u=1,2, \ldots, n)$ and $\mathscr{L}_{u}^{\prime}=\left\langle\left\langle u_{j}^{\prime}, \eta_{u}^{\prime}\right\rangle,\left\langle\Lambda_{u}^{\prime}, \lambda_{u}^{\prime}\right\rangle\right\rangle$ $(j=1,2, \ldots, n)$ be two collections of CIFNs and $p, q \geq 0, k>0$. If $\Upsilon_{u} \geq \Upsilon_{u}^{\prime}, \eta_{u} \geq \eta_{u}^{\prime}, \Lambda_{u} \geq \Lambda_{u}^{\prime}$, and $\lambda_{u} \geq \lambda_{u}^{\prime}$ for all $\left.j=1,2, \ldots, n\right)$, then

$$
\operatorname{CIFWDBM}^{p, q}\left(\mathscr{L}_{1}, \mathscr{L}_{2}, \ldots, \mathscr{L}_{n}\right) \geq \operatorname{CIFWDBM}^{p, q}\left(\mathscr{L}_{1}^{\prime}, \mathscr{L}_{2}^{\prime}, \ldots, \mathscr{L}_{n}^{\prime}\right)
$$

Boundedness 2: let $\mathscr{L}_{u}=\left\langle\left\langle\Upsilon_{u}, \eta_{u}\right\rangle,\left\langle\Lambda_{u}, \lambda_{u}\right\rangle\right\rangle$

$$
\mathscr{L}^{-} \leq \operatorname{CIFWDBM}{ }^{p, q}\left(\mathscr{L}_{1}, \mathscr{L}_{2}, \ldots, \mathscr{L}_{n}\right) \leq \mathscr{L}^{+}
$$
$(u=1,2, \ldots, n)$ be CIFNs and $p, q \geq 0, k>0$, and $\mathscr{L}^{+}=\left\langle\left\langle\max \Upsilon_{u}, \max \eta_{u}\right\rangle, \quad\left\langle\min \Lambda_{u}, \min \lambda_{u}\right\rangle\right\rangle$, $\mathscr{L}^{-}=\left\langle\left\langle\min _{u}^{u} \Upsilon_{u}, \min _{u}^{u} \eta_{u}\right\rangle, \quad\left\langle\max _{u}^{u} \Lambda_{u}, \max _{u}^{u} \lambda_{u}\right\rangle\right\rangle$. Then, $\left.\left.\max _{u}^{u} \Lambda_{u}, \max _{u}^{u} \lambda_{u}\right\rangle\right\rangle$. Definition

9. Let $\mathscr{L}_{u}=\left\langle\left\langle\Upsilon_{u}, \eta_{u}\right\rangle,\left\langle\Lambda_{u}, \lambda_{u}\right\rangle\right\rangle$ $(u=1,2, \ldots, n)$ be CIFNs and $p, q \geq 0, k>0$. Then, a 
mapping CIFDGBM ${ }^{p, q}: \mathscr{L}^{n} \longrightarrow \mathscr{L}$ is called CIF Dombi geometric Bonferroni mean operator if

$$
\operatorname{CIFDGBM}^{p, q}\left(\mathscr{L}_{1}, \mathscr{L}_{2}, \ldots, \mathscr{L}_{n}\right)=\left(\frac{1}{n(n-1)} \sum_{\substack{u, v=1 \\ u \neq v}}^{n}\left(\mathscr{E}_{u} \mathscr{L}_{u}\right)^{p} \oplus_{\mathscr{D}}\left(\mathscr{E}_{v} \mathscr{L}_{v}\right)^{q}\right)^{1 /(p+q)}
$$

Theorem 3. Let $\mathscr{L}_{u}=\left\langle\left\langle Y_{u}, \eta_{u}\right\rangle,\left\langle\Lambda_{u}, \lambda_{u}\right\rangle\right\rangle\left(u=1,2\right.$, aggregated values by using the CIFDGBM ${ }^{p, q}$ operator is a $\ldots, n)$ be CIFNs and $p, q \geq 0, k>0$. Then, the resultant CIFN, and

$$
\begin{aligned}
& \operatorname{CIFDGBM}^{p, q}\left(\mathscr{L}_{1}, \mathscr{L}_{2}, \ldots, \mathscr{L}_{n}\right)
\end{aligned}
$$

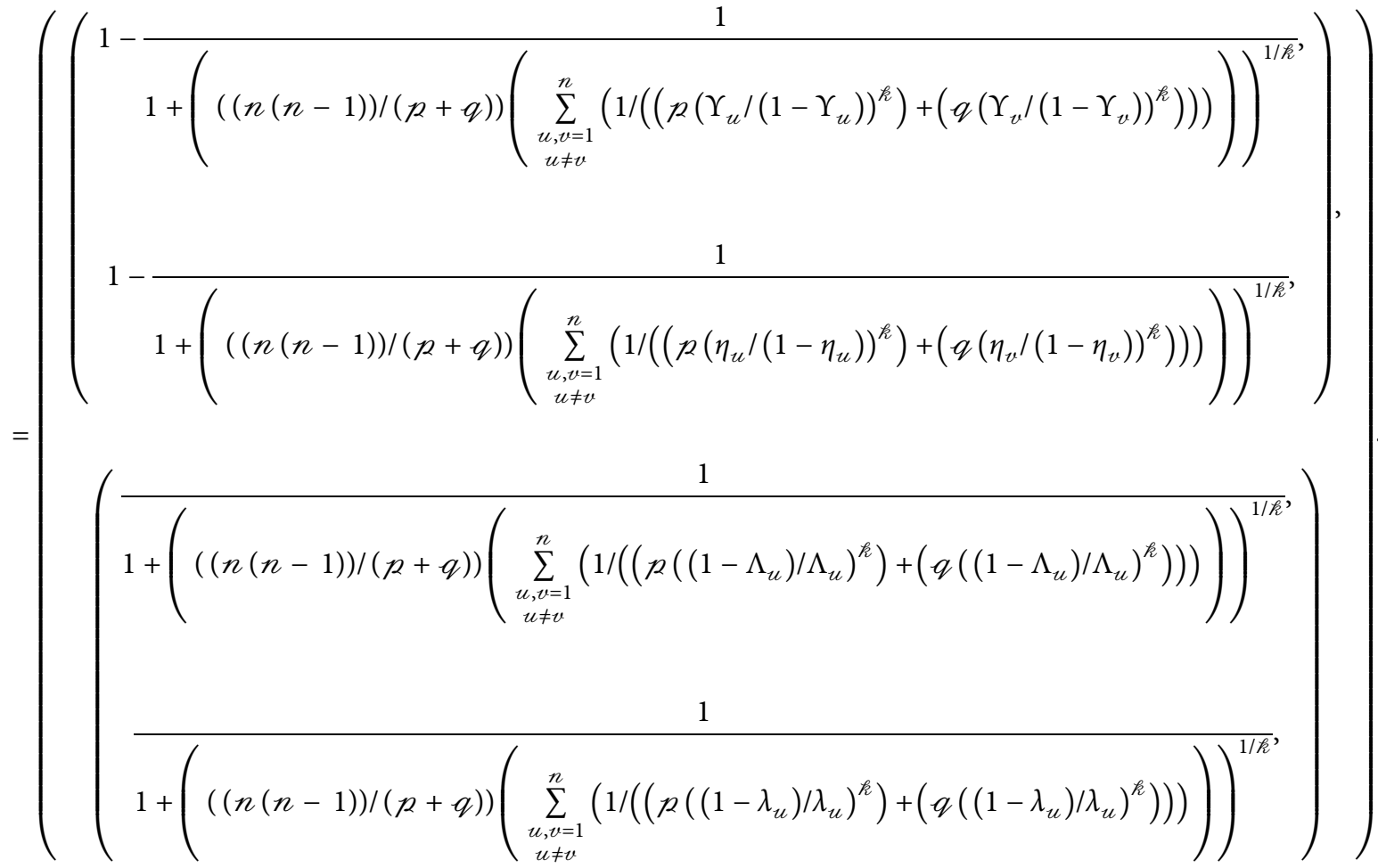

We present some desirable properties for CIFDGBMp,q operator as follows:

Idempotency 3: let $\mathscr{L}_{u}=\left\langle\left\langle\Upsilon_{u}, \eta_{u}\right\rangle,\left\langle\Lambda_{u}, \lambda_{u}\right\rangle\right\rangle$ $(u=1,2, \ldots, n)$ be CIFNs and $p, q \geq 0, k>0$. If $\mathscr{L}_{1}=\mathscr{L}_{2}=\ldots=\mathscr{L}_{n}$, that is, $\mathscr{L}_{u}=\mathscr{L}=\langle\langle Y, \eta\rangle$, $\langle\Lambda, \lambda\rangle\rangle$, then

$$
\operatorname{CIFDGBM}^{p, q}\left(\mathscr{L}_{1}, \mathscr{L}_{2}, \ldots, \mathscr{L}_{n}\right)=\mathscr{L} .
$$

Monotonicity 3: let $\mathscr{L}_{u}=\left\langle\left\langle\Upsilon_{u}, \eta_{u}\right\rangle,\left\langle\Lambda_{u}, \lambda_{u}\right\rangle\right\rangle$ and $\mathscr{L}_{u}^{\prime}=\left\langle\left\langle Y_{u}^{\prime}, \eta_{j}^{\prime}\right\rangle,\left\langle\Lambda_{u}^{\prime}, \lambda_{u}^{\prime}\right\rangle\right\rangle(u=1,2, \ldots, n)$ be two collections of CIFNs and $p, q \geq 0, k>0$. If $\Upsilon_{u} \geq \Upsilon_{u}^{\prime}$, $\eta_{u} \geq \eta_{u}^{\prime}, \Lambda_{u} \geq \Lambda_{u}^{\prime}$, and $\lambda_{u} \geq \lambda_{u}^{\prime}$ for all $\left.u=1,2, \ldots, n\right)$, then
$\operatorname{CIFDGBM}^{p, q}\left(\mathscr{L}_{1}, \mathscr{L}_{2}, \ldots, \mathscr{L}_{n}\right) \geq \operatorname{CIFDGBM}^{p, q}$

$$
\left(\mathscr{L}_{1}^{\prime}, \mathscr{L}_{2}^{\prime}, \ldots, \mathscr{L}_{n}^{\prime}\right)
$$

Boundedness 3: let $\mathscr{L}_{u}=\left\langle\left\langle Y_{u}, \eta_{u}\right\rangle,\left\langle\Lambda_{u}, \lambda_{u}\right\rangle\right\rangle(u=$ $1,2, \ldots, n) b e$ CIFNs and $p, q \geq 0, k>0$, and $\mathscr{L}^{+}=$ $\left\langle\left\langle\max \Upsilon_{u}, \max \eta_{u}\right\rangle, \quad\left\langle\min \Lambda_{u}, \min \lambda_{u}\right\rangle\right\rangle, \quad \mathscr{L}^{-}=$ $\left\langle\left\langle\min _{u}^{u} \Upsilon_{u}, \min _{u}^{u} \eta_{u}\right\rangle,\left\langle\max _{u}^{u} \Lambda_{u}, \max _{u}^{u} \lambda_{u}\right\rangle\right\rangle$. Then,

$\mathscr{L}^{-} \leq \operatorname{CIFDGBM}^{p, q}\left(\mathscr{L}_{1}, \mathscr{L}_{2}, \ldots, \mathscr{L}_{n}\right) \leq \mathscr{L}^{+}$.

Definition 10. Let $\mathscr{L}_{u}=\left\langle\left\langle\Upsilon_{u}, \eta_{u}\right\rangle,\left\langle\Lambda_{u}, \lambda_{u}\right\rangle\right\rangle(u=1,2$, $\ldots, n)$ be CIFNs and $p, q \geq 0, k>0$. Then, a mapping 
CIFWDGBM $^{p, q}: \mathscr{L}^{n} \longrightarrow \mathscr{L}$ is called a complex intuitionistic fuzzy weighted Dombi Bonferroni mean operator if

$$
\operatorname{CIFWDGBM}^{p, q}\left(\mathscr{L}_{1}, \mathscr{L}_{2}, \ldots, \mathscr{L}_{n}\right)=\left(\frac{1}{n(n-1)} \sum_{\substack{u, v=1 \\ u \neq v}}^{n}\left(\mathscr{E}_{u} \mathscr{L}_{u}\right)^{p} \oplus_{\mathscr{D}}\left(\mathscr{E}_{v} \mathscr{L}_{v}\right)^{q}\right)^{1 /(p+q)},
$$

where $\mathscr{E}=\left(\mathscr{E}_{1}, \mathscr{E}_{2} \ldots, \mathscr{E}_{n}\right)^{T}$ is a weighted vector of $\mathscr{L}_{u}=$ $\left\langle\left\langle\Upsilon_{u}, \eta_{u}\right\rangle,\left\langle\Lambda_{u}, \lambda_{u}\right\rangle\right\rangle(u=1,2, \ldots, n) \quad$ such that $1 \geq \mathscr{E}_{n} \geq 0, \sum_{u=1}^{n} \mathscr{E}_{u}=1$.
Theorem 4. Let $\mathscr{L}_{u}=\left\langle\left\langle\Upsilon_{u}, \eta_{u}\right\rangle,\left\langle\Lambda_{u}, \lambda_{u}\right\rangle\right\rangle(u=1,2$, $\ldots, n)$ beCIFNs and $p, q \geq 0, k>0$. Then by using the CIFWDGBM ${ }^{2, q}$ operators, the resultant aggregated value is a CIFN and

$\operatorname{CIFWDGBM}^{p, q}\left(\mathscr{L}_{1}, \mathscr{L}_{2}, \ldots, \mathscr{L}_{n}\right)$

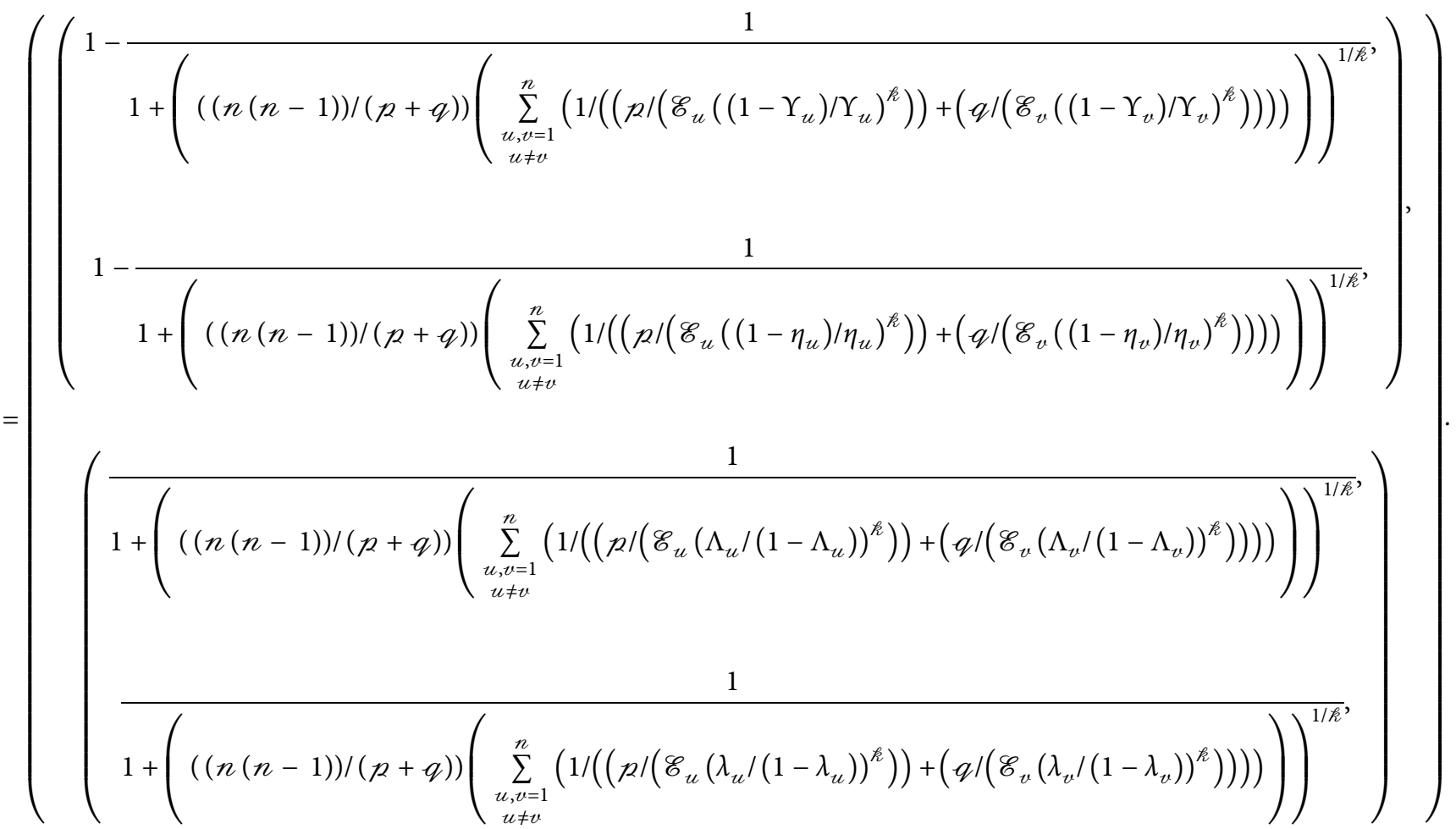

where $\mathscr{E}=\left(\mathscr{E}_{1}, \mathscr{E}_{2} \ldots, \mathscr{E}_{n}\right)^{T}$ is a weighted vector of $\mathscr{L}_{u}=$ $\left\langle\left\langle\Upsilon_{u}, \eta_{u}\right\rangle,\left\langle\Lambda_{u}, \lambda_{u}\right\rangle\right\rangle(u=1,2, \ldots, n) \quad$ such that $1 \geq \mathscr{E}_{n} \geq 0, \sum_{u=1}^{n} \mathscr{E}_{u}=1$.

We present some desirable properties for CIFWDGBM operator as follows:

Idempotency 4: let $\mathscr{L}_{u}=\left\langle\left\langle\Upsilon_{u}, \eta_{u}\right\rangle,\left\langle\Lambda_{u}, \lambda_{u}\right\rangle\right\rangle$ $(u=1,2, \ldots, n)$ be CIFNs and $p, q \geq 0, k>0$. If $\mathscr{L}_{1}=\mathscr{L}_{2}=\ldots=\mathscr{L}_{n}$, that is, $\mathscr{L}_{j}=\mathscr{L}=\langle\langle\Upsilon, \eta\rangle$, $\langle\Lambda, \lambda\rangle\rangle$, then

$$
\operatorname{CIFWDGBM}^{2, q}\left(\mathscr{L}_{1}, \mathscr{L}_{2}, \ldots, \mathscr{L}_{n}\right)=\mathscr{L} \text {. }
$$

Monotonicity 4: let $\mathscr{L}_{u}=\left\langle\left\langle\Upsilon_{u}, \eta_{u}\right\rangle,\left\langle\Lambda_{u}, \lambda_{u}\right\rangle\right\rangle$ $(u=1,2, \ldots, n)$ and $\quad \mathscr{L}_{j}^{\prime}=\left\langle\left\langle\Upsilon_{j}^{\prime}, \eta_{j}^{\prime}\right\rangle,\left\langle\Lambda_{j}^{\prime}, \lambda_{j}^{\prime}\right\rangle\right\rangle$ $(j=1,2, \ldots, n)$ be two collections of CIFNs, $p, q \geq$ $0, k>0$. If $\Upsilon_{u} \geq \Upsilon_{u}^{\prime}, \eta_{u} \geq \eta_{u}^{\prime}, \Lambda_{u} \geq \Lambda_{u}^{\prime}$, and $\lambda_{u} \geq \lambda_{u}^{\prime}$ for all $u=1,2, \ldots, n)$, then

$$
\begin{aligned}
& \operatorname{CIFWDGBM}^{p, q}\left(\mathscr{L}_{1}, \mathscr{L}_{2}, \ldots, \mathscr{L}_{n}\right) \\
& \geq \operatorname{CIFWDGBM}^{p, q}\left(\mathscr{L}_{1}^{\prime}, \mathscr{L}_{2}^{\prime}, \ldots, \mathscr{L}_{n}^{\prime}\right) .
\end{aligned}
$$

Boundedness 4: let $\mathscr{L}_{u}=\left\langle\left\langle\Upsilon_{u}, \eta_{u}\right\rangle,\left\langle\Lambda_{u}, \lambda_{u}\right\rangle\right\rangle$ $(u=1,2, \ldots, n)$ beCIFNs and $p, q \geq 0, k>0$, and 
$\mathscr{L}^{+}=\left\langle\left\langle\max \Upsilon_{u}, \max \eta_{u}\right\rangle,\left\langle\min \Lambda_{u}, \min \lambda_{u}\right\rangle\right\rangle, \mathscr{L}^{-}$ $=\left\langle\left\langle\min _{u} Y_{u}, \min _{u} \eta_{u}^{u}\right\rangle,\left\langle\max _{u} \Lambda_{u}^{u}, \max _{u} \lambda_{u}^{u}\right\rangle\right\rangle$. Then,

$\mathscr{L}^{-} \leq \operatorname{CIFWDGBM}^{p, q}\left(\mathscr{L}_{1}, \mathscr{L}_{2}, \ldots, \mathscr{L}_{n}\right) \leq \mathscr{L}^{+}$.

\section{MCGDM Approach Based on CIFDBM Operators}

In this section, a MCGDM method is proposed based on CIFDBM operators. The advantages of the proposed operators are that it not only considers interrelationships between criteria but are also more flexible in the decisionmaking process.

Generally, the MCGDM problem involves data and knowledge that is vague and imprecise. We consider MCGDM problems in which all criteria values expressed in CIFNs and interrelationships between decision-making criteria or decision-makers' preferences are considered. The collection of expert viewpoints is critical in MCGDM problems in order to properly conduct the assessment process. Based on CIFDBM operators in the following, a MCGDM approach is proposed under CIFNs where the assessment values are aggregated by utilizing the CIFDBM operators:

Step 1. Since for each alternative $\mathscr{A} \ell_{u}(u=1,2, \ldots, m)$, each $\operatorname{DM}_{r}(r=1,2, \ldots, t)$ is invited to express their individual evaluation or preference according to each criterion $\mathscr{C}_{v}(u=1,2, \ldots, n)$ by CIFNs $\mathscr{L}_{u v}^{(r)}=\left\langle\left\langle Y_{u v}^{(r)}\right.\right.$, $\left.\left.\eta_{u v}^{(r)}\right\rangle,\left\langle\Lambda_{u v}^{(r)}, \quad \lambda_{u v}^{(r)}\right\rangle\right\rangle(u=1,2, \ldots, m ; v=1,2, \ldots n ;$ $\mu=1,2, . ., t)$. Then, we obtain a CIF MCGDM matrix as follows:

Let " $\Omega^{(\varkappa)}=\left[\mathscr{L}_{u v}^{(r)}\right]_{m \times n}$ be an CIF decision matrix, provided by decision makers $\operatorname{DM}_{r}(r=1,2, \ldots, t)$, as the following form:

\begin{tabular}{ccccc}
\hline & $\mathcal{C r}_{1}$ & $\mathcal{C r}_{2}$ & $\ldots$ & $\mathcal{C r}_{n}$ \\
\hline $\mathcal{A l} \ell_{1}$ & $\mathcal{L}_{11}^{(t)}$ & $\mathcal{L}_{12}^{(t)}$ & $\ldots$ & $\mathcal{L}_{1 n}^{(t)}$ \\
$\mathcal{A l} \ell_{2}$ & $\mathcal{L}_{21}^{(t)}$ & $\mathcal{L}_{22}^{(t)}$ & $\ldots$ & $\mathcal{L}_{2 n}^{(t)}$ \\
$\vdots$ & $\vdots$ & $\vdots$ & $\ddots$ & $\vdots$ \\
$\mathcal{A l} \ell_{m}$ & $\mathcal{L}_{m 1}^{(t)}$ & $\mathcal{L}_{m 2}^{(t)}$ & $\cdots$ & $\mathcal{L}_{m n}^{(t)}$ \\
\hline
\end{tabular}

where $\quad \mathscr{L}_{u v}^{(\varkappa)}=\left\langle\left\langle\Upsilon_{u v}^{(r)}, \eta_{u v}^{(\varkappa)}\right\rangle,\left\langle\Lambda_{u v}^{(r)}, \lambda_{u v}^{(\varkappa)}\right\rangle\right\rangle(u=$ $1,2, \ldots, m ; v=1,2, \ldots n ; \mu=1,2, . ., t)$ is the CIFN representing the alternative's efficiency ranking $\mathscr{A} l_{u} \in \mathscr{A l}$ with respect to the criteria $\mathscr{C} \mu_{u} \in \mathscr{C} r$ provided by the DMs $D M_{r} \in D M$.
Step 2. Aggregating the assessment information provided by the DMs by means of CIFDWBM/ CIFDWGBM operator.

$$
\begin{aligned}
& \operatorname{CIFWDBM}^{p, q}\left(\mathscr{L}_{1}, \mathscr{L}_{2}, \ldots, \mathscr{L}_{n}\right)
\end{aligned}
$$

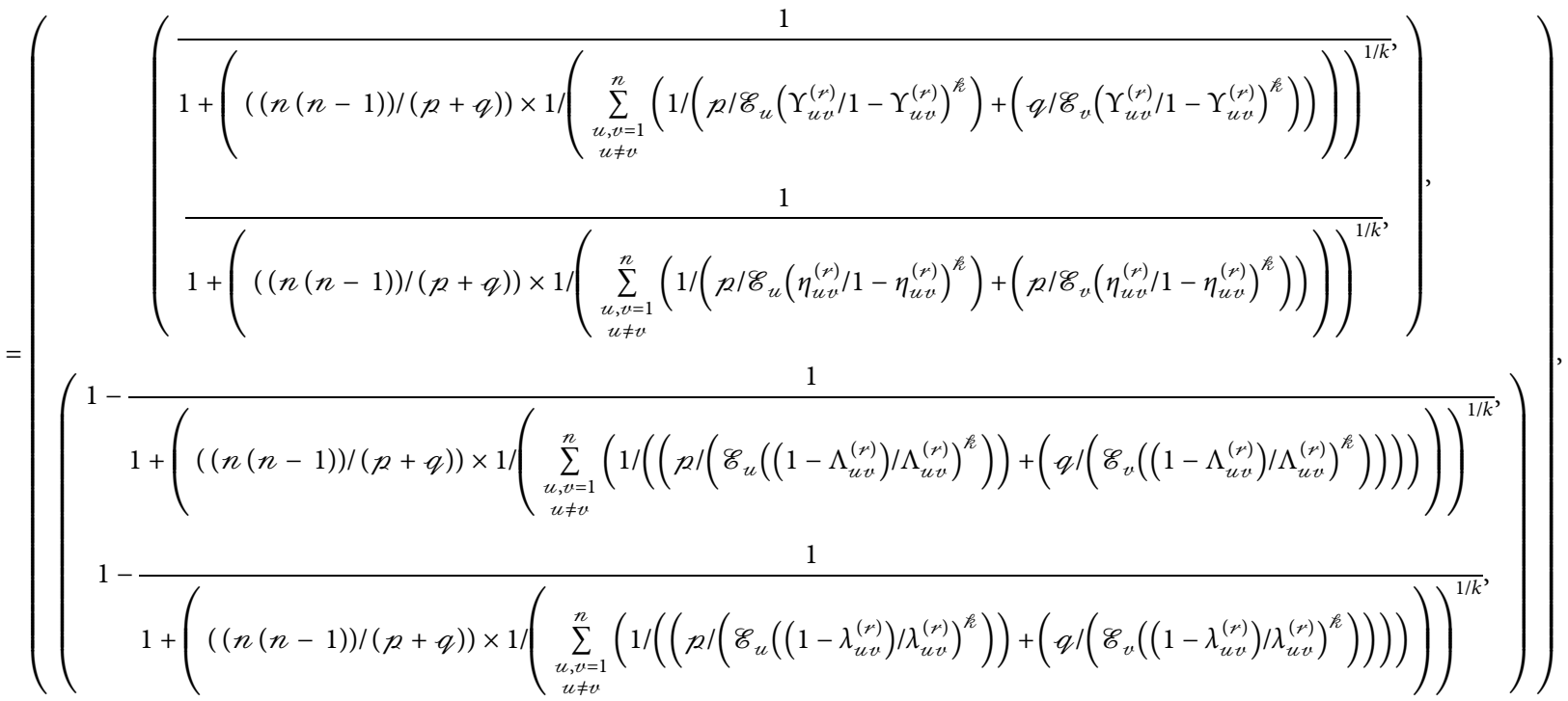


or

$\operatorname{CIFWDGBM}^{p, q}\left(\mathscr{L}_{1}, \mathscr{L}_{2}, \ldots, \mathscr{L}_{n}\right)$

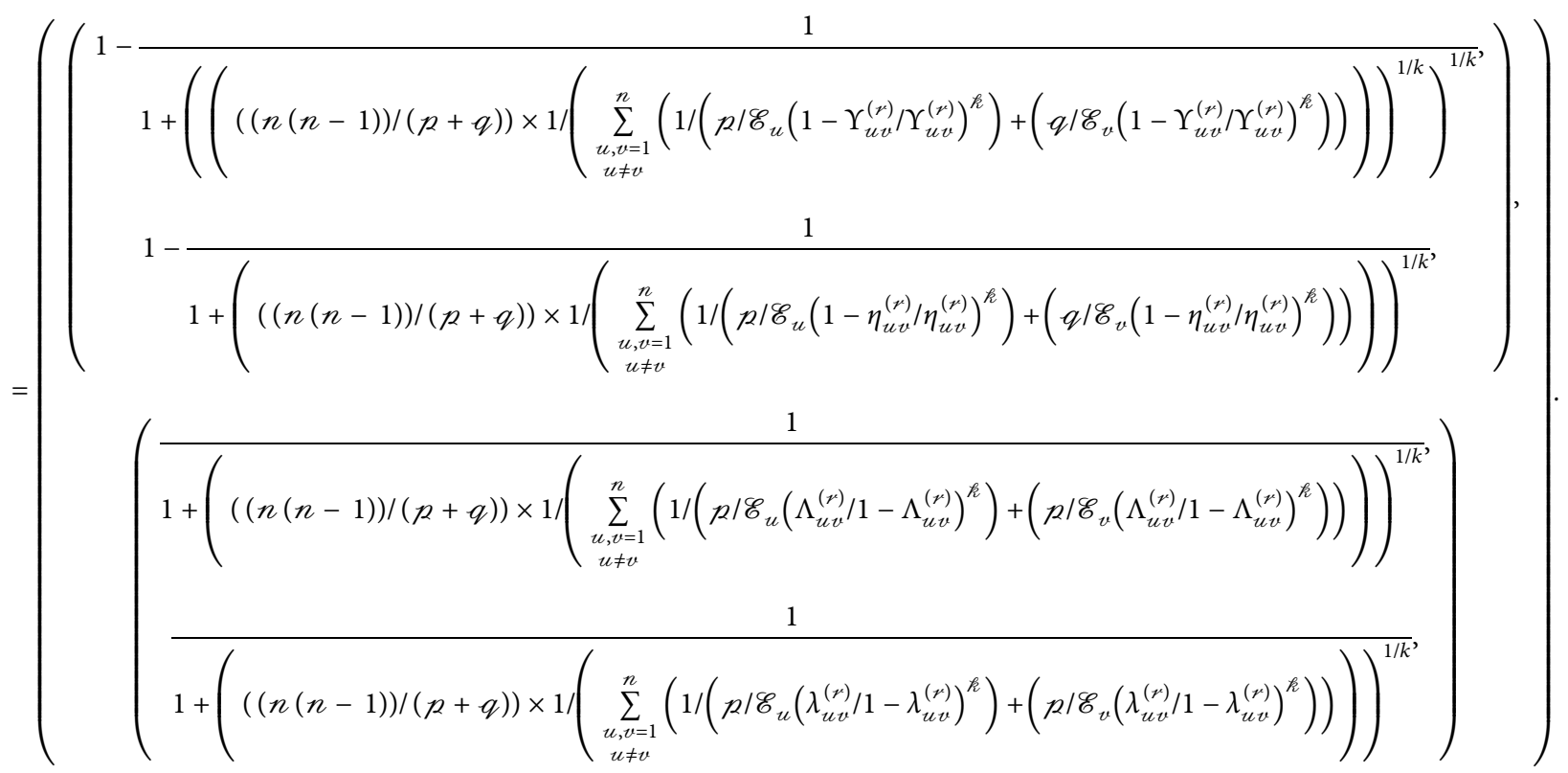

Step 3. Utilize the entropy measure defined in equation (4) to find the attribute weight of the assessment information.
Step 4. Aggregate the total assessment information by utilizing the CIFDWBM/CIFDWGBM operator.

$\operatorname{CIFWDBM}^{R, q}\left(\mathscr{L}_{1}, \mathscr{L}_{2}, \ldots, \mathscr{L}_{n}\right)$

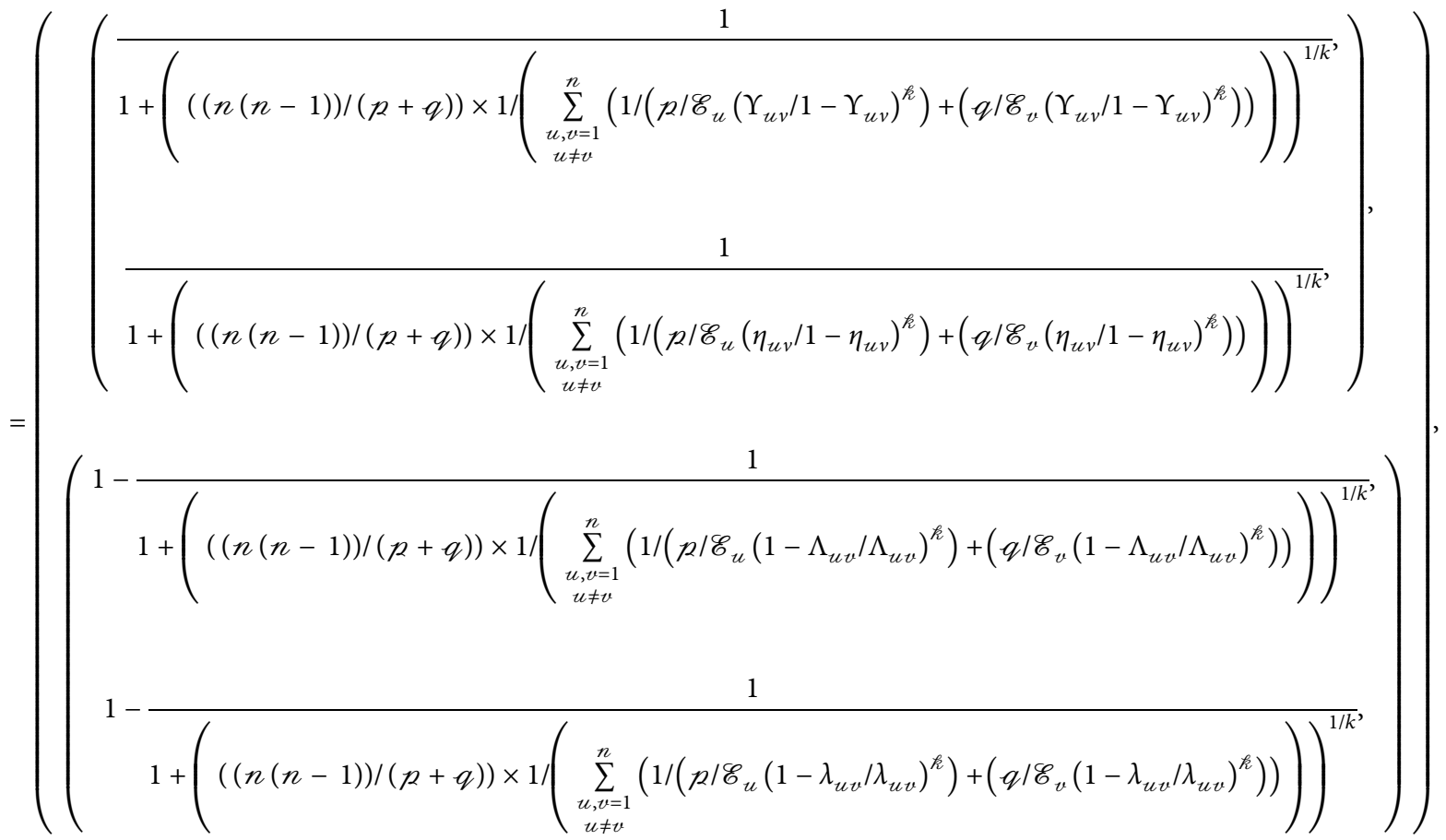


or

$$
\begin{aligned}
& \operatorname{CIFWDGBM}^{p, q}\left(\mathscr{L}_{1}, \mathscr{L}_{2}, \ldots, \mathscr{L}_{n}\right)
\end{aligned}
$$

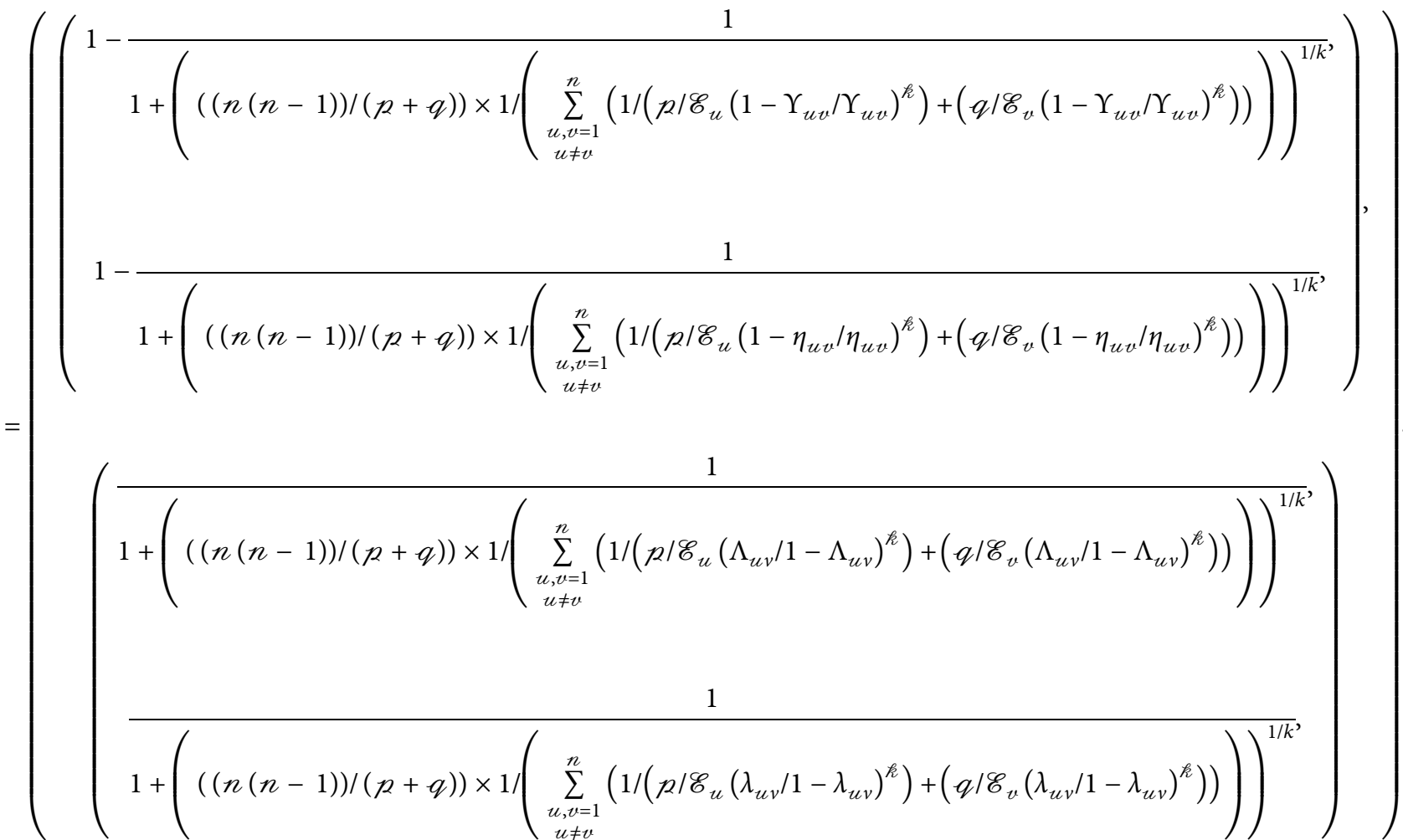

Step 5. Rank the alternatives by calculating the score values of the CIFNs and then select the best alternative.

\section{Illustrative Example}

Due to the rapid advancement of computer and information technology, information has become a new commodity for businesses and has become increasingly relevant. In recent years, it is the need of all companies together that how to secure information security, which is a big problem. Therefore, in this section, we take a numerical example that how to evaluate the enterprise's information security management. We look at an issue of information security management assessment in a particular organization. Let $\mathrm{DM}_{1}, \mathrm{DM}_{2}$, and $\mathrm{DM}_{3}$ be three experts that have been invited from some famous universities and play a role of DMs. The DMs select five large state-owned enterprises and are denoted by $\mathscr{A} \ell_{1}$, $\mathscr{A} \ell_{2}, \mathscr{A} \ell_{3}, \mathscr{A} \ell_{4}$, and $\mathscr{A} \ell_{5}$. Meanwhile, they describe four characteristics based on the previous study denoted as $\mathscr{C} \mu_{1}$, $\mathscr{C} r_{2}, \mathscr{C} r_{3}$, and $\mathscr{C} r_{4}$, which are presented as follows:

$\mathscr{C} r_{1}=$ control of physical protection (it includes the security management of facilities and the security management of the environment)
$\mathscr{C} \boldsymbol{r}_{2}=$ control of operational protection (it includes stuff-like operating system security management, network security management, and database security management)

$\mathscr{C} r_{3}=$ risk management (environment risk, process risk, database risk, and control risk are all included.)

$\mathscr{C} \boldsymbol{r}_{4}=$ human resource and policy management (it includes human resource management, strategy management, and applicable policy management for the organization, among other things).

The three experts presented their preference values in the form of CIFNs as presented in Tables 1-3.

Expert weight is calculated using the method presented by $\mathrm{Xu}[35]$.

Step 1. Using the CIFWDBM/CIFDGBM operator to aggregate the assessment information presented by the DMs. We obtain the collective CIF decision matrices presented in Table 4.

Step 2. Compute the attribute weight by using the CIF entropy measure presented in equation (4), and we get the attribute weight as 
TABLE 1: Complex intuitionistic fuzzy decision matrix " $\Omega^{(1)}=\left[\mathscr{L}_{u v}^{(1)}\right]_{5 \times 4}$.

\begin{tabular}{lllll}
\hline & $\mathscr{C} r_{1}$ & $\mathscr{C} r_{2}$ & $\mathscr{C} r_{3}$ & $\mathscr{C} r_{4}$ \\
\hline $\mathscr{A} \ell_{1}$ & $\left\langle 0.5 e^{i 2 \pi 0.4}, 0.3 e^{i 2 \pi 0.1}\right\rangle$ & $\left\langle 0.3 e^{i 2 \pi 0.4}, 0.2 e^{i 2 \pi 0.3}\right\rangle$ & $\left\langle 0.3 e^{i 2 \pi 0.3}, 0.3 e^{i 2 \pi 0.4}\right\rangle$ & $\left\langle 0.5 e^{i 2 \pi 0.4}, 0.2 e^{i 2 \pi 0.3}\right\rangle$ \\
$\mathscr{A} \ell_{2}$ & $\left\langle 0.2 e^{i 2 \pi 0.3}, 0.4 e^{i 2 \pi 0.2}\right\rangle$ & $\left\langle 0.4 e^{i 2 \pi 0.2}, 0.2 e^{i 2 \pi 0.4}\right\rangle$ & $\left\langle 0.1 e^{i 2 \pi 0.4}, 0.6 e^{i 2 \pi 0.3}\right\rangle$ & $\left\langle 0.3 e^{i 2 \pi 0.2}, 0.4 e^{i 2 \pi 0.4}\right\rangle$ \\
$\mathscr{A} \ell_{3}$ & $\left\langle 0.3 e^{i 2 \pi 0.2}, 0.5 e^{i 2 \pi 0.4}\right\rangle$ & $\left\langle 0.2 e^{i 2 \pi 0.3}, 0.3 e^{i 2 \pi 0.4}\right\rangle$ & $\left\langle 0.5 e^{i 2 \pi 0.3}, 0.3 e^{i 2 \pi 0.2}\right\rangle$ & $\left\langle 0.3 e^{i 2 \pi 0.4}, 0.2 e^{i 2 \pi 0.2}\right\rangle$ \\
$\mathscr{A} \ell_{4}$ & $\left\langle 0.4 e^{i 2 \pi 0.4}, 0.3 e^{i 2 \pi 0.2}\right\rangle$ & $\left\langle 0.5 e^{i 2 \pi 0.3}, 0.2 e^{i 2 \pi 0.2}\right\rangle$ & $\left\langle 0.3 e^{i 2 \pi 0.2}, 0.2 e^{i 2 \pi 0.1}\right\rangle$ & $\left\langle 0.1 e^{i 2 \pi 0.4}, 0.6 e^{i 2 \pi 0.1}\right\rangle$ \\
$\mathscr{A} \ell_{5}$ & $\left\langle 0.6 e^{i 2 \pi 0.3}, 0.2 e^{i 2 \pi 0.1}\right\rangle$ & $\left\langle 0.3 e^{i 2 \pi 0.2}, 0.4 e^{i 2 \pi 0.3}\right\rangle$ & $\left\langle 0.5 e^{i 2 \pi 0.1}, 0.1 e^{i 2 \pi 0.4}\right\rangle$ & $\left\langle 0.2 e^{i 2 \pi 0.3}, 0.4 e^{i 2 \pi 0.2}\right\rangle$ \\
\hline
\end{tabular}

TABLE 2: Complex intuitionistic fuzzy decision matrix " $\Omega^{(2)}=\left[\mathscr{L}_{u v}^{(2)}\right]_{5 \times 4}$.

\begin{tabular}{ccccc}
\hline & $\mathscr{C} r_{1}$ & $\mathscr{C} r_{2}$ & $\mathscr{C} r_{3}$ & $\mathscr{C} r_{4}$ \\
\hline $\mathscr{A} \ell_{1}$ & $\left\langle 0.3 e^{i 2 \pi 0.2}, 0.5 e^{i 2 \pi 0.4}\right\rangle$ & $\left\langle 0.2 e^{i 2 \pi 0.3}, 0.3 e^{i 2 \pi 0.4}\right\rangle$ & $\left\langle 0.5 e^{i 2 \pi 0.3}, 0.3 e^{i 2 \pi 0.2}\right\rangle$ & $\left\langle 0.3 e^{i 2 \pi 0.4}, 0.2 e^{i 2 \pi 0.2}\right\rangle$ \\
$\mathscr{A} e_{2}$ & $\left\langle 0.6 e^{i 2 \pi 0.3}, 0.2 e^{i 2 \pi 0.1}\right\rangle$ & $\left\langle 0.3 e^{i 2 \pi 0.2}, 0.4 e^{i 2 \pi 0.3}\right\rangle$ & $\left\langle 0.5 e^{i 2 \pi 0.1}, 0.1 e^{i 2 \pi 0.4}\right\rangle$ & $\left\langle 0.2 e^{i 2 \pi 0.3}, 0.4 e^{i 2 \pi 0.2}\right\rangle$ \\
$\mathscr{A} \ell_{3}$ & $\left\langle 0.5 e^{i 2 \pi 0.4}, 0.3 e^{i 2 \pi 0.1}\right\rangle$ & $\left\langle 0.3 e^{i 2 \pi 0.4}, 0.2 e^{i 2 \pi 0.3}\right\rangle$ & $\left\langle 0.3 e^{i 2 \pi 0.3}, 0.3 e^{i 2 \pi 0.4}\right\rangle$ & $\left\langle 0.5 e^{i 2 \pi 0.4}, 0.2 e^{i 2 \pi 0.3}\right\rangle$ \\
$\mathscr{A} e_{4}$ & $\left\langle 0.2 e^{i 2 \pi 0.3}, 0.4 e^{i 2 \pi 0.2}\right\rangle$ & $\left\langle 0.4 e^{i 2 \pi 0.2}, 0.2 e^{i 2 \pi 0.4}\right\rangle$ & $\left\langle 0.1 e^{i 2 \pi 0.4}, 0.6 e^{i 2 \pi 0.3}\right\rangle$ & $\left\langle 0.3 e^{i 2 \pi 0.2}, 0.4 e^{i 2 \pi 0.4}\right\rangle$ \\
$\mathscr{A} e_{5}$ & $\left\langle 0.4 e^{i 2 \pi 0.4}, 0.3 e^{i 2 \pi 0.2}\right\rangle$ & $\left\langle 0.5 e^{i 2 \pi 0.3}, 0.2 e^{i 2 \pi 0.2}\right\rangle$ & $\left\langle 0.3 e^{i 2 \pi 0.2}, 0.2 e^{i 2 \pi 0.1}\right\rangle$ & $\left\langle 0.1 e^{i 2 \pi 0.4}, 0.6 e^{i 2 \pi 0.1}\right\rangle$ \\
\hline
\end{tabular}

TABLE 3: Complex intuitionistic fuzzy decision matrix " $\Omega^{(3)}=\left[\mathscr{L}_{u v}^{(3)}\right]_{5 \times 4}$.

\begin{tabular}{lllll}
\hline & $\mathscr{C} r_{1}$ & $\mathscr{C} r_{2}$ & $\mathscr{C} r_{3}$ & $\mathscr{C} r_{4}$ \\
\hline $\mathscr{A} \ell_{1}$ & $\left(0.2 e^{i 2 \pi 0.3}, 0.3 e^{i 2 \pi 0.4}\right)$ & $\left(0.5 e^{i 2 \pi 0.3}, 0.3 e^{i 2 \pi 0.2}\right)$ & $\left(0.3 e^{i 2 \pi 0.2}, 0.5 e^{i 2 \pi 0.4}\right)$ & $\left(0.5 e^{i 2 \pi 0.4}, 0.3 e^{i 2 \pi 0.1}\right)$ \\
$\mathscr{A} \ell_{2}$ & $\left(0.3 e^{i 2 \pi 0.2}, 0.4 e^{i 2 \pi 0.3}\right)$ & $\left(0.5 e^{i 2 \pi 0.1}, 0.1 e^{i 2 \pi 0.4}\right)$ & $\left(0.6 e^{i 2 \pi 0.3}, 0.2 e^{i 2 \pi 0.1}\right)$ & $\left(0.2 e^{i 2 \pi 0.3}, 0.4 e^{i 2 \pi 0.2}\right)$ \\
$\mathscr{A} \ell_{3}$ & $\left(0.3 e^{i 2 \pi 0.4}, 0.2 e^{i 2 \pi 0.3}\right)$ & $\left(0.3 e^{i 2 \pi 0.3}, 0.3 e^{i 2 \pi 0.4}\right)$ & $\left(0.5 e^{i 2 \pi 0.4}, 0.3 e^{i 2 \pi 0.1}\right)$ & $\left(0.3 e^{i 2 \pi 0.2}, 0.5 e^{i 2 \pi 0.4}\right)$ \\
$\mathscr{A} \ell_{4}$ & $\left(0.4 e^{i 2 \pi 0.2}, 0.2 e^{i 2 \pi 0.4}\right)$ & $\left(0.1 e^{i 2 \pi 0.4}, 0.6 e^{i 2 \pi 0.3}\right)$ & $\left(0.2 e^{i 2 \pi 0.3}, 0.4 e^{i 2 \pi 0.2}\right)$ & $\left(0.4 e^{i 2 \pi 0.4}, 0.3 e^{i 2 \pi 0.2}\right)$ \\
$\mathscr{A} \ell_{5}$ & $\left(0.5 e^{i 2 \pi 0.3}, 0.2 e^{i 2 \pi 0.2}\right)$ & $\left(0.3 e^{i 2 \pi 0.2}, 0.2 e^{i 2 \pi 0.1}\right)$ & $\left(0.4 e^{i 2 \pi 0.4}, 0.3 e^{i 2 \pi 0.2}\right)$ & $\left(0.6 e^{i 2 \pi 0.3}, 0.2 e^{i 2 \pi 0.1}\right)$ \\
\hline
\end{tabular}

TABle 4: Collective complex intuitionistic fuzzy decision matrix " $\Omega=\left[\mathscr{L}_{u v}\right]_{5 \times 4}$.

\begin{tabular}{|c|c|c|c|c|}
\hline & $\mathscr{C} r_{1}$ & $\mathscr{C} r_{2}$ & $\mathscr{C} r_{3}$ & $\mathscr{C} r_{4}$ \\
\hline $\mathscr{A} \ell_{1}$ & $\begin{array}{c}\left(0.2056 e^{i 2 \pi 0.1818}\right. \\
\left.0.5085 e^{i 2 \pi 0.4672}\right)\end{array}$ & $\begin{array}{c}\left(0.1929 e^{i 2 \pi 0.2210}\right. \\
\left.0.3967 e^{i 2 \pi 0.4312}\right)\end{array}$ & $\begin{array}{c}\left(0.2232 e^{i 2 \pi 0.1669}\right. \\
\left.0.5153 e^{i 2 \pi 0.5150}\right)\end{array}$ & $\begin{array}{l}\left(0.2953 e^{i 2 \pi 0.2774},\right. \\
\left.0.3594 e^{i 2 \pi 0.3089}\right)\end{array}$ \\
\hline $\mathscr{A l} \ell_{2}$ & $\begin{array}{c}\left(0.1981 e^{i 2 \pi 0.1669}\right. \\
\left.0.5140 e^{i 2 \pi 0.3356}\right)\end{array}$ & $\begin{array}{l}\left(0.2062 e^{i 2 \pi 0.1154}\right. \\
\left.0.4036 e^{i 2 \pi 0.5277}\right)\end{array}$ & $\begin{array}{l}\left(0.3958 e^{i 2 \pi 0.1302}\right. \\
\left.0.3335 e^{i 2 \pi 0.3292}\right)\end{array}$ & $\begin{array}{l}\left(0.1451 e^{i 2 \pi 0.2210},\right. \\
\left.0.4837 e^{i 2 \pi 0.3131}\right)\end{array}$ \\
\hline $\mathscr{A} \ell_{3}$ & $\begin{array}{l}\left(0.2232 e^{i 2 \pi 0.2214}\right. \\
\left.0.4723 e^{i 2 \pi 0.4467}\right)\end{array}$ & $\begin{array}{l}\left(0.1703 e^{i 2 \pi 0.2144}\right. \\
\left.0.4149 e^{i 2 \pi 0.5287}\right)\end{array}$ & $\begin{array}{l}\left(0.2953 e^{i 2 \pi 0.2208}\right. \\
\left.0.4386 e^{i 2 \pi 0.3292}\right)\end{array}$ & $\begin{array}{l}\left(0.2232 e^{i 2 \pi 0.2114},\right. \\
\left.0.4101 e^{i 2 \pi 0.4343}\right)\end{array}$ \\
\hline $\mathscr{A} \ell_{4}$ & $\begin{array}{l}\left(0.2392 e^{i 2 \pi 0.1884}\right. \\
\left.0.4312 e^{i 2 \pi 0.3913}\right)\end{array}$ & $\begin{array}{l}\left(0.2242 e^{i 2 \pi 0.1844}\right. \\
\left.0.4204 e^{i 2 \pi 0.4387}\right)\end{array}$ & $\begin{array}{l}\left(0.1075 e^{i 2 \pi 0.1853}\right. \\
\left.0.5724 e^{i 2 \pi 0.3140}\right)\end{array}$ & $\begin{array}{l}\left(0.1717 e^{i 2 \pi 0.2094},\right. \\
\left.0.5861 e^{i 2 \pi 0.3499}\right)\end{array}$ \\
\hline $\mathscr{A} \ell_{5}$ & $\begin{array}{l}\left(0.3582 e^{i 2 \pi 0.2144}\right. \\
\left.0.3489 e^{i 2 \pi 0.2654}\right)\end{array}$ & $\begin{array}{l}\left(0.2232 e^{i 2 \pi 0.1392}\right. \\
\left.0.3960 e^{i 2 \pi 0.3089}\right)\end{array}$ & $\begin{array}{l}\left(0.2681 e^{i 2 \pi 0.1277}\right. \\
\left.0.3005 e^{i 2 \pi 0.3629}\right)\end{array}$ & $\begin{array}{l}\left(0.1154 e^{i 2 \pi 0.2144},\right. \\
\left.0.5497 e^{i 2 \pi 0.2164}\right)\end{array}$ \\
\hline
\end{tabular}

$$
\begin{aligned}
& v_{1}=0.2653, \\
& v_{2}=0.2480, \\
& v_{3}=0.2711, \\
& v_{4}=0.2156 .
\end{aligned}
$$

Step 4. Utilizing equations (80) and (81) aggregate to overall CIFNs, and we get the final aggregated values as

$$
\begin{aligned}
& \mathfrak{\mathfrak { A }}_{1}=\left(0.1265 e^{i 2 \pi 0.1148}, 0.6191 e^{i 2 \pi 0.6073}\right), \\
& \mathfrak{\mathfrak { A }}_{2}=\left(0.1163 e^{i 2 \pi 0.0819}, 0.6116 e^{i 2 \pi 0.5443}\right), \\
& \mathfrak{\mathfrak { A }}_{3}=\left(0.1239 e^{i 2 \pi 0.1213}, 0.5768 e^{i 2 \pi 0.6130}\right), \\
& \mathfrak{\mathfrak { A }}_{4}=\left(0.1060 e^{i 2 \pi 0.1058}, 0.6744 e^{i 2 \pi 0.5465}\right), \\
& \mathfrak{\mathfrak { A }}_{5}=\left(0.1260 e^{i 2 \pi 0.0912}, 0.5699 e^{i 2 \pi 0.4481}\right) .
\end{aligned}
$$


TABLE 5: Collective complex intuitionistic fuzzy decision matrix " $\Omega=\left[\mathscr{L}_{u v}\right]_{5 \times 4}$.

\begin{tabular}{|c|c|c|c|c|}
\hline & $\mathscr{C} r_{1}$ & $\mathscr{C} r_{2}$ & $\mathscr{C} r_{3}$ & $\mathscr{C} r_{4}$ \\
\hline $\mathscr{A} \ell_{1}$ & $\begin{array}{l}\left(0.4462 e^{i 2 \pi 0.4358}\right. \\
\left.0.2088 e^{i 2 \pi 0.1788}\right)\end{array}$ & $\begin{array}{l}\left(0.4840 e^{i 2 \pi 0.4645}\right. \\
\left.0.1600 e^{i 2 \pi 0.1704}\right)\end{array}$ & $\begin{array}{l}\left(0.5056 e^{i 2 \pi 0.3877}\right. \\
\left.0.2314 e^{i 2 \pi 0.2111}\right)\end{array}$ & $\begin{array}{l}\left(0.5987 e^{i 2 \pi 0.5365}\right. \\
\left.0.1409 e^{i 2 \pi 0.1126}\right)\end{array}$ \\
\hline $\mathscr{A} l_{2}$ & $\begin{array}{l}\left(0.5081 e^{i 2 \pi 0.3877}\right. \\
\left.0.2111 e^{i 2 \pi 0.1116}\right)\end{array}$ & $\begin{array}{l}\left(0.4531 e^{i 2 \pi 0.2932}\right. \\
\left.0.1450 e^{i 2 \pi 0.2433}\right)\end{array}$ & $\begin{array}{l}\left(0.6701 e^{i 2 \pi 0.3829}\right. \\
\left.0.1064 e^{i 2 \pi 0.1031}\right)\end{array}$ & $\begin{array}{l}\left(0.3430 e^{i 2 \pi 0.4645}\right. \\
\left.0.2027 e^{i 2 \pi 0.1206}\right)\end{array}$ \\
\hline$A l_{3}$ & $\begin{array}{l}\left(0.4944 e^{i 2 \pi 0.4741}\right. \\
\left.0.2008 e^{i 2 \pi 0.1471}\right)\end{array}$ & $\begin{array}{l}\left(0.6130 e^{i 2 \pi 0.4643}\right. \\
\left.0.1667 e^{i 2 \pi 0.2448}\right)\end{array}$ & $\begin{array}{l}\left(0.5987 e^{i 2 \pi 0.4690}\right. \\
\left.0.1904 e^{i 2 \pi 0.1031}\right)\end{array}$ & $\begin{array}{l}\left(0.5056 e^{i 2 \pi 0.4770}\right. \\
\left.0.1582 e^{i 2 \pi 0.1808}\right)\end{array}$ \\
\hline $\mathscr{A} \ell_{4}$ & $\begin{array}{l}\left(0.5149 e^{i 2 \pi 0.4027}\right. \\
\left.0.1735 e^{i 2 \pi 0.1523}\right)\end{array}$ & $\begin{array}{c}\left(0.4959 e^{i 2 \pi 0.4473}\right. \\
\left.0.1612 e^{i 2 \pi 0.1709}\right)\end{array}$ & $\begin{array}{l}\left(0.3093 e^{i 2 \pi 0.4319}\right. \\
\left.0.2264 e^{i 2 \pi 0.1002}\right)\end{array}$ & $\begin{array}{l}\left(0.3916 e^{i 2 \pi 0.4960}\right. \\
\left.0.2847 e^{i 2 \pi 0.1040}\right)\end{array}$ \\
\hline $\mathscr{A} \ell_{5}$ & $\begin{array}{l}\left(0.6456 e^{i 2 \pi 0.5357}\right. \\
\left.0.1312 e^{i 2 \pi 0.0914}\right)\end{array}$ & $\begin{array}{l}\left(0.5056 e^{i 2 \pi 0.3446}\right. \\
\left.0.1511 e^{i 2 \pi 0.1126}\right)\end{array}$ & $\begin{array}{l}\left(0.5469 e^{i 2 \pi 0.3150}\right. \\
\left.0.1079 e^{i 2 \pi 0.1107}\right)\end{array}$ & $\begin{array}{l}\left(0.3850 e^{i 2 \pi 0.5357}\right. \\
\left.0.2257 e^{i 2 \pi 0.0714}\right)\end{array}$ \\
\hline
\end{tabular}

Step 5. Utilizing equation (2), we get the score values as follows:

$$
\begin{aligned}
& \mathcal{S}\left(\mathscr{A} \ell_{1}\right)=-0.9850, \\
& \mathcal{S}\left(\mathscr{A} \ell_{2}\right)=-0.9577, \\
& \mathcal{S}(\mathscr{A} \ell)_{3}=-0.9445, \\
& \mathcal{S}\left(\mathscr{A} \ell_{4}\right)=-1.0090, \\
& \mathcal{S}(\mathscr{A} \ell)_{5}=-0.8008 .
\end{aligned}
$$

Based on score values, the ranking of alternatives is

$$
\mathscr{A} l_{5}>\mathscr{A} l_{3}>\mathscr{A} l_{2}>\mathscr{A} l_{1}>\mathscr{A} l_{4} \text {. }
$$

Hence, the most desirable alternative is $\mathscr{A} \ell_{5}$. For CIFWDGBM operator

Step 1'. Using the CIFWDGBM operator to aggregate the assessment information presented by the DMs. We obtain the collective CIF decision matrices presented in Table 5

Step 2'. Compute the attribute weight by using the CIF entropy measure presented in equation (4), and we get the attribute weight as

$$
\begin{aligned}
& v_{1}=0.2498, \\
& v_{2}=0.2218, \\
& v_{3}=0.2350, \\
& v_{4}=0.2934 .
\end{aligned}
$$

Step 3'. Utilizing equations (80) and (81) aggregate to overall CIFNs, and we get the final aggregated values as

$$
\begin{aligned}
& \mathfrak{U}_{1}^{\prime}=\left(0.6773 e^{i 2 \pi 0.6292}, 0.0989 e^{i 2 \pi 0.0887}\right), \\
& \mathfrak{U}_{2}^{\prime}=\left(0.6560 e^{i 2 \pi 0.5556}, 0.0859 e^{i 2 \pi 0.0692}\right), \\
& \mathfrak{U}_{3}^{\prime}=\left(0.7116 e^{i 2 \pi 0.6412}, 0.0965 e^{i 2 \pi 0.0865}\right), \\
& \mathfrak{A}_{4}^{\prime}=\left(0.6054 e^{i 2 \pi 0.6169}, 0.1118 e^{i 2 \pi 0.0677}\right), \\
& \mathfrak{U}_{5}^{\prime}=\left(0.6974 e^{i 2 \pi 0.6090}, 0.0768 e^{i 2 \pi 0.0495}\right) .
\end{aligned}
$$

Step 4'. Utilizing equation (2), we get the score values as follows:

$$
\begin{aligned}
& \mathcal{S}\left(\mathscr{A} \ell_{1}^{\prime}\right)=1.1189, \\
& \mathcal{S}\left(\mathscr{A} \ell_{2}^{\prime}\right)=1.0564, \\
& \mathcal{S}\left(\mathscr{A} \ell_{3}^{\prime}\right)=1.1699, \\
& \mathcal{S}\left(\mathscr{A} \ell_{4}^{\prime}\right)=1.0428, \\
& \mathcal{S}\left(\mathscr{A} \ell_{5}^{\prime}\right)=1.1801 .
\end{aligned}
$$

Based on score values the ranking of alternatives is

$$
\mathscr{A} l_{5}>\mathscr{A l} l_{3}>\mathscr{A l} l_{2}>\mathscr{A} l_{1}>\mathscr{A} l_{4} \text {. }
$$

Hence, the most desirable alternative is $\mathscr{A} \ell_{5}$.

5.1. Impact of Different Parameters $p, q$, and Delta on the Ranking of Alternatives. Since the above result is based on the parameters $p, q$, and delta having fixed values. Tables 6 and 7 show a more detailed overview of the effect of the various parameters by adjusting the parameters $p, q$, and $k$. It is seen that corresponding to $p=1, q=1$ and $p=1$ $q=2, p=2, q=1, p=2, q=2$, the order of the alternatives is $\mathscr{A} \ell_{5}>\mathscr{A} l_{3}>\mathscr{A} l_{2}>\mathscr{A} l_{1}>\mathscr{A} l_{4}$. However, to increase the value of $\delta=2$ to $\delta=3$, the score values of the alternative tend to increase. Also, we get the same score values for, $p=1, q=1$ and $p=1, q=2, p=2, q=1, p=2, q=2$ when $k=2$ to $k=3$. Thus, for different values of parameters, the DMs can pick the nature of the problem.

5.2. Comparison Analysis. In this section, we looked at how the proposed findings are compared to all of the current comparisons in the CIFS environment. To compare and describe the proposed method's success with a number of current CIFS studies, a comparative study is conducted, as presented in Table 8 .

From Table 8 , we see that we get the same best alternatives by applying the existing methods and proposed operators. The ranking of alternatives by using the CIFWA/ CIFWG operator [33], CIFEWA/CIFFWG operator [34], 
TABLE 6: Ranking of alternatives for different parameters by using the CIFDWBM operator.

\begin{tabular}{|c|c|c|c|c|c|c|c|}
\hline \multirow{2}{*}{$k$} & \multirow{2}{*}{ Values of $p$ and $q$} & \multicolumn{5}{|c|}{ Score values } & \multirow{2}{*}{ Ranking of alternatives } \\
\hline & & $\mathcal{S}\left(\mathscr{A} \ell_{1}\right)$ & $\mathcal{S}\left(\mathscr{A} \ell_{2}\right)$ & $\mathcal{S}\left(\mathscr{A} \ell_{3}\right)$ & $\mathcal{S}\left(\mathscr{A} \ell_{4}\right)$ & $\mathcal{S}\left(\mathscr{A} \ell_{5}\right)$ & \\
\hline \multirow{4}{*}{$k=2$} & $\begin{array}{l}p=1 \\
q=1\end{array}$ & -0.9850 & -0.9577 & -0.9445 & -1.0090 & -0.8008 & $\mathscr{A} l_{5}>\mathscr{A} l_{3}>\mathscr{A} l_{2}>\mathscr{A} l_{1}>\mathscr{A} l_{4}$ \\
\hline & $\begin{array}{l}p=1 \\
q=2\end{array}$ & -0.9798 & -0.9500 & -0.9378 & -1.0055 & -0.7948 & $\mathscr{A} \ell_{5}>\mathscr{A} l_{3}>\mathscr{A} l_{2}>\mathscr{A} \ell_{1}>\mathscr{A} \ell_{4}$ \\
\hline & $\begin{array}{l}p=2 \\
q=1\end{array}$ & -0.9789 & -0.9500 & -0.9378 & -1.0039 & -0.7933 & $\mathscr{A} l_{5}>\mathscr{A} l_{3}>\mathscr{A} l_{2}>\mathscr{A} l_{1}>\mathscr{A} l_{4}$ \\
\hline & $\begin{array}{l}p=2 \\
q=2\end{array}$ & -0.9850 & -0.9577 & -0.9445 & -1.0090 & -0.8008 & $\mathscr{A} l_{5}>\mathscr{A} l_{3}>\mathscr{A} l_{2}>\mathscr{A} l_{1}>\mathscr{A} l_{4}$ \\
\hline \multirow{4}{*}{$k=2.5$} & $\begin{array}{l}p=1 \\
q=1\end{array}$ & -0.8910 & -0.8633 & -0.8487 & -0.9147 & -0.7001 & $\mathscr{A} l_{5}>\mathscr{A} l_{3}>\mathscr{A} l_{2}>\mathscr{A} \ell_{1}>\mathscr{A} l_{4}$ \\
\hline & $\begin{array}{l}p=1 \\
q=2\end{array}$ & -0.8845 & -0.8552 & -0.8411 & -0.9061 & -0.6938 & $\mathscr{A} l_{5}>\mathscr{A} l_{3}>\mathscr{A} l_{2}>\mathscr{A} l_{1}>\mathscr{A} l_{4}$ \\
\hline & $\begin{array}{l}p=2 \\
q=1\end{array}$ & -0.8845 & -0.8552 & -0.8411 & -0.9096 & -0.6923 & $\mathscr{A} l_{5}>\mathscr{A} l_{3}>\mathscr{A} l_{2}>\mathscr{A} \ell_{1}>\mathscr{A} l_{4}$ \\
\hline & $\begin{array}{l}p=2 \\
q=2\end{array}$ & -0.8910 & -0.8633 & -0.8487 & -0.9147 & -0.7001 & $\mathscr{A} e_{5}>\mathscr{A} l_{3}>\mathscr{A} l_{2}>\mathscr{A} l_{1}>\mathscr{A} l_{4}$ \\
\hline \multirow{4}{*}{$k=3$} & $\begin{array}{l}p=1 \\
q=1\end{array}$ & -0.8264 & -0.7972 & -0.7830 & -0.8480 & -0.6292 & $\mathscr{A} l_{5}>\mathscr{A} l_{3}>\mathscr{A} l_{2}>\mathscr{A} l_{1}>\mathscr{A} l_{4}$ \\
\hline & $\begin{array}{l}p=1 \\
q=2\end{array}$ & -0.8190 & -0.7892 & -0.7748 & -0.8374 & -0.6227 & $\mathscr{A} l_{5}>\mathscr{A} l_{3}>\mathscr{A} l_{2}>\mathscr{A} l_{1}>\mathscr{A} l_{4}$ \\
\hline & $\begin{array}{l}p=2 \\
q=1\end{array}$ & -0.8190 & -0.7892 & -0.7748 & -0.8430 & -0.6211 & $\mathscr{A} l_{5}>\mathscr{A} l_{3}>\mathscr{A} l_{2}>\mathscr{A} l_{1}>\mathscr{A} l_{4}$ \\
\hline & $\begin{array}{l}p=2 \\
q=2\end{array}$ & -0.8264 & -0.7972 & -0.7830 & -0.8480 & -0.6292 & $\mathscr{A} l_{5}>\mathscr{A} l_{3}>\mathscr{A} l_{2}>\mathscr{A} l_{1}>\mathscr{A} l_{4}$ \\
\hline
\end{tabular}

TABLE 7: Ranking of alternatives for different parameters by using the CIFDWGBM operator.

\begin{tabular}{|c|c|c|c|c|c|c|c|}
\hline \multirow{2}{*}{$k$} & \multirow{2}{*}{ Values of $p$ and $q$} & \multicolumn{5}{|c|}{ Score values } & \multirow{2}{*}{ Ranking of alternatives } \\
\hline & & $\mathcal{S}\left(\mathscr{A} \ell_{1}\right)$ & $\mathcal{S}\left(\mathscr{A} l_{2}\right)$ & $\mathcal{S}\left(\mathscr{A} \ell_{3}\right)$ & $\mathcal{S}\left(\mathscr{A} \ell_{4}\right)$ & $\mathcal{S}\left(\mathscr{A} \ell_{5}\right)$ & \\
\hline \multirow{4}{*}{$k=2$} & $\begin{array}{l}p=1 \\
q=1\end{array}$ & 1.1189 & 1.0564 & 1.1699 & 1.0428 & 1.1801 & $\mathscr{A} \ell_{5}>\mathscr{A} l_{3}>\mathscr{A} \ell_{2}>\mathscr{A} l_{1}>\mathscr{A} l_{4}$ \\
\hline & $\begin{array}{l}p=1 \\
q=2\end{array}$ & 1.1159 & 1.0479 & 1.1674 & 1.0375 & 1.1719 & $\mathscr{A} \ell_{5}>\mathscr{A} \ell_{3}>\mathscr{A} \ell_{2}>\mathscr{A} \ell_{1}>\mathscr{A} \ell_{4}$ \\
\hline & $\begin{array}{l}p=2 \\
q=1\end{array}$ & 1.1159 & 1.0479 & 1.1659 & 1.0375 & 1.1719 & $\mathscr{A} \ell_{5}>\mathscr{A} \ell_{3}>\mathscr{A} \ell_{2}>\mathscr{A} \ell_{1}>\mathscr{A} \ell_{4}$ \\
\hline & $\begin{array}{l}p=2 \\
q=2\end{array}$ & 1.1190 & 1.0564 & $1 . .1691$ & 1.0428 & 1.8101 & $\mathscr{A} \ell_{5}>\mathscr{A} \ell_{3}>\mathscr{A} \ell_{2}>\mathscr{A} \ell_{1}>\mathscr{A} \ell_{4}$ \\
\hline \multirow{4}{*}{$k=2.5$} & $\begin{array}{l}p=1 \\
q=1\end{array}$ & 1.0309 & $0 . .9702$ & 1.0822 & 0.9531 & 1.0992 & $\mathscr{A} \ell_{5}>\mathscr{A} \ell_{3}>\mathscr{A} \ell_{2}>\mathscr{A} \ell_{1}>\mathscr{A} \ell_{4}$ \\
\hline & $\begin{array}{l}p=1 \\
q=2\end{array}$ & 1.0271 & 0.9602 & 1.0801 & 0.9472 & 1.0905 & $\mathscr{A} \ell_{5}>\mathscr{A} \ell_{3}>\mathscr{A} \ell_{2}>\mathscr{A} \ell_{1}>\mathscr{A} \ell_{4}$ \\
\hline & $\begin{array}{l}p=2 \\
q=1\end{array}$ & 1.0271 & 0.9633 & 1.0787 & 0.9472 & 1.0905 & $\mathscr{A} \ell_{5}>\mathscr{A} \ell_{3}>\mathscr{A} \ell_{2}>\mathscr{A} \ell_{1}>\mathscr{A} \ell_{4}$ \\
\hline & $\begin{array}{l}p=2 \\
q=2\end{array}$ & 1.0309 & 0.9702 & 1.0822 & 0.9531 & 1.0992 & $\mathscr{A} \ell_{5}>\mathscr{A} \ell_{3}>\mathscr{A} \ell_{2}>\mathscr{A} \ell_{1}>\mathscr{A} \ell_{4}$ \\
\hline \multirow{4}{*}{$k=3$} & $\begin{array}{l}p=1 \\
q=1\end{array}$ & 0.9691 & 0.9103 & 1.0208 & 0.8906 & 1.0408 & $\mathscr{A} \ell_{5}>\mathscr{A} \ell_{3}>\mathscr{A} \ell_{2}>\mathscr{A} \ell_{1}>\mathscr{A} \ell_{4}$ \\
\hline & $\begin{array}{l}p=1 \\
q=2\end{array}$ & 0.9974 & 0.8996 & 1.0184 & 0.8842 & 1.0321 & $\mathscr{A} \ell_{5}>\mathscr{A} \ell_{3}>\mathscr{A} \ell_{2}>\mathscr{A} \ell_{1}>\mathscr{A} \ell_{4}$ \\
\hline & $\begin{array}{l}p=2 \\
q=1\end{array}$ & 0.9647 & 0.9032 & 1.0172 & 0.8842 & 1.0321 & $\mathscr{A} l_{5}>\mathscr{A} l_{3}>\mathscr{A} l_{2}>\mathscr{A} l_{1}>\mathscr{A} l_{4}$ \\
\hline & $\begin{array}{l}p=2 \\
q=2\end{array}$ & 0.9691 & 0.9103 & 1.0208 & 0.8906 & 1.0408 & $\mathscr{A} l_{5}>\mathscr{A} l_{3}>\mathscr{A} l_{2}>\mathscr{A} l_{1}>\mathscr{A} l_{4}$ \\
\hline
\end{tabular}

and CIFLWA operator [36] is $\mathscr{A} \ell_{5}>\mathscr{A} \ell_{3}>\mathscr{A} \ell_{2}>\mathscr{A} \ell_{1}$ $>A \ell_{4}$. Also, by using proposed operators, we have the same ranking of alternatives as $\mathscr{A} \ell_{5}>\mathscr{A} \ell_{3}>\mathscr{A} \ell_{2}>\mathscr{A} \ell_{1}>\mathscr{A} \ell_{4}$.
However, there is no interrelationship among the criteria by using existing operators. But in the proposed method, there is an interrelationship among the criteria. Moreover, by 
TABLE 8: Analysis result of comparative studies of the proposed operators with existing methods.

\begin{tabular}{|c|c|c|c|c|c|c|}
\hline Operator & $\mathcal{S}\left(\mathscr{A} \ell_{1}\right)$ & $\mathcal{S}\left(\mathscr{A} \ell_{2}\right)$ & $\delta\left(A \ell_{3}\right)$ & $\mathcal{S}\left(\mathscr{A} \ell_{4}\right)$ & $\mathcal{S}\left(\mathscr{A} \ell_{5}\right)$ & Ranking of alternatives \\
\hline CIFWBM operator [19] & -1.5088 & -1.4849 & -1.4820 & -1.5211 & -1.4095 & $\mathscr{A} \ell_{5}>\mathscr{A} \ell_{3}$ \\
\hline CIFWA operator [34] & -0.4387 & -0.3872 & -0.3685 & -0.4865 & -0.2424 & $\mathscr{A} l_{5}>\mathscr{A} \ell_{3}>\mathscr{A} l_{2}>\mathscr{A} l_{1}>\mathscr{A} \ell_{4}$ \\
\hline CIFWG operator [34] & -0.4682 & -0.4422 & -0.3123 & -0.5138 & -0.2942 & $\mathscr{A} e_{5}>\mathscr{A} e_{3}>\mathscr{A} l_{2}>\mathscr{A} l_{1}>\mathscr{A} \ell_{4}$ \\
\hline CIFEWA operator [34] & -0.4433 & -0.3955 & -0.3725 & -0.4446 & -0.2492 & $\mathscr{A} l_{5}>\mathscr{A} l_{3}>\mathscr{A} l_{2}>\mathscr{A} l_{1}>\mathscr{A} l_{4}$ \\
\hline CIFEWG operator [34] & -0.4641 & -0.4349 & -0.3909 & -0.5102 & -0.2873 & $\mathscr{A} \ell_{5}>\mathscr{A} e_{3}>\mathscr{A} l_{2}>\mathscr{A} l_{1}>\mathscr{A} \ell_{4}$ \\
\hline CIFLWA operator [36] & -0.6671 & -0.7751 & -0.7144 & -0.7408 & -0.8818 & $\mathscr{A} \ell_{5}>\mathscr{A} \ell_{3}>\mathscr{A} \ell_{2}>\mathscr{A} l_{1}>\mathscr{A} \ell_{4}$ \\
\hline CIFDWBM operator & -0.9850 & -0.9577 & -0.9445 & -1.0090 & -0.8008 & $\mathscr{A} l_{5}>\mathscr{A} l_{3}>\mathscr{A} l_{2}>\mathscr{A} l_{1}>\mathscr{A} \ell_{4}$ \\
\hline CIFDWGBM operator & 1.1189 & 1.0564 & 1.1699 & 1.0428 & 1.1801 & $\mathscr{A} l_{5}>\mathscr{A} l_{3}>\mathscr{A} l_{2}>\mathscr{A} l_{1}>\mathscr{A} l_{4}$ \\
\hline
\end{tabular}

using the CIFWBM operator [19], we get the ranking of alternatives as $\mathscr{A} \ell_{5}>\mathscr{A} \ell_{3}>\mathscr{A} \ell_{2}>\mathscr{A} \ell_{1}>\mathscr{A} \ell_{4}$, which is the same as obtained by using the proposed operators. However, the existing CIFWBM operator only has an interrelationship among the criteria but there is no flexibility, while the proposed operators not only have an interrelationship among the criteria but also have more flexibility as compared to existing operators. Hence, the present method is more reliable and valid as compared with the existing methods.

\section{Conclusion and Further Studies}

Since Bonferroni mean operators are very effective tools to discuss interrelation among the criteria. Also, CIFS is more suitable in order to deal with the phase term as well as the amplitude term during the decision-making process. Therefore, keeping the advantages of the CIFS and Bonferroni mean operators, in this paper, we developed some new aggregation operators based on Dombi T-norm and Tconorm with Bonferroni mean operators under the CIFS environment. We proposed a CIFDBM operator and a CIFDGBM operator and their weighted forms for CIFNs. Moreover, some desirable properties of the proposed operators are discussed and investigated in different cases. Further, we proposed an MCGDM approach based on the developed operators. Furthermore, to show the application and effectiveness of the developed approach, we presented a real-world decision-making problem of the evaluation of information security management. Finally, we compared the proposed approach with existing methods and showed that our proposed approach is more effective as compared to existing ones. In the future, we will develop some more generalized interactive aggregation operators by using various fuzzy environments [37,38].

\section{Data Availability}

No data were used to support this study.

\section{Conflicts of Interest}

The authors have no conflicts of interest.

\section{References}

[1] L. A. Zadeh, "Fuzzy sets," Information and Control, vol. 8, no. 3, pp. 338-353, 1965.
[2] K. T. Atanassov, "Intuitionistic fuzzy sets," Fuzzy Sets and Systems, vol. 20, no. 1, pp. 87-96, 1986.

[3] F. E. Boran and D. Akay, "A biparametric similarity measure on intuitionistic fuzzy sets with applications to pattern recognition," Information Sciences, vol. 255, pp. 45-57, 2014.

[4] S. M. Chen, "Similarity measures between vague sets and between elements," IEEE Transactions on Systems, Man, and Cybernetics, Part B (Cybernetics), vol. 27, no. 1, pp. 153-158, 1997.

[5] W.-L. Hung and M.-S. Yang, "Similarity measures of intuitionistic fuzzy sets based on Hausdorff distance," Pattern Recognition Letters, vol. 25, no. 14, pp. 1603-1611, 2004.

[6] L. Dengfeng and C. Chuntian, "New similarity measures of intuitionistic fuzzy sets and application to pattern recognitions," Pattern Recognition Letters, vol. 23, no. 1-3, pp. 221-225, 2002.

[7] W. Jiang, B. Wei, X. Liu, X. Li, and H. Zheng, "Intuitionistic fuzzy power aggregation operator based on entropy and its application in decision making," International Journal of Intelligent Systems, vol. 33, no. 1, pp. 49-67, 2018.

[8] Y. Song, X. Wang, W. Quan, and W. Huang, "A new approach to construct similarity measure for intuitionistic fuzzy sets," Soft Computing, vol. 23, no. 6, pp. 1985-1998, 2017.

[9] P. Liu, J. Liu, and S. M. Chen, "Some intuitionistic fuzzy Dombi Bonferroni mean operators and their application to multi-attribute group decision making," Journal of the Operational Research Society, vol. 69, 2017.

[10] C. Bonferroni, "Sulle medie multiple di potenze," Bolletino Matematica Italiana, vol. 5, no. 3-4, pp. 267-270, 1950.

[11] J. Dombi, "A general class of fuzzy operators, the demorgan class of fuzzy operators and fuzziness measures induced by fuzzy operators," Fuzzy Sets and Systems, vol. 8, no. 2, pp. 149-163, 1982.

[12] M. S. A. Khan, S. Abdullah, A. Ali, and F. Amin, "Pythagorean fuzzy prioritized aggregation operators and their application to multi-attribute group decision making," Granular Computing, vol. 4, no. 2, pp. 249-263, 2019.

[13] M. S. Ali Khan, S. Abdullah, and A. Ali, "Multiattribute group decision-making based on Pythagorean fuzzy Einstein prioritized aggregation operators," International Journal of Intelligent Systems, vol. 34, no. 5, pp. 1001-1033, 2018.

[14] M. Sajjad Ali Khan, S. Abdullah, M. Yousaf Ali, I. Hussain, and M. Farooq, "Extension of TOPSIS method base on Choquet integral under interval-valued Pythagorean fuzzy environment," Journal of Intelligent and Fuzzy Systems, vol. 34, no. 1, pp. 267-282, 2018.

[15] M. S. A. Khan and S. Abdullah, "Interval-valued Pythagorean fuzzy GRA method for multiple-attribute decision making with incomplete weight information," International Journal of Intelligent Systems, vol. 33, no. 8, pp. 1689-1716, 2018. 
[16] M. S. A. Khan, S. Abdullah, A. Ali, N. Siddiqui, and F. Amin, "Pythagorean hesitant fuzzy sets and their application to group decision making with incomplete weight information," Journal of Intelligent and Fuzzy Systems, vol. 33, no. 6, pp. 3971-3985, 2017.

[17] M. Sajjad Ali Khan, A. Ali, S. Abdullah, F. Amin, and F. Hussain, "New extension of TOPSIS method based on Pythagorean hesitant fuzzy sets with incomplete weight information," Journal of Intelligent and Fuzzy Systems, vol. 35, no. 5, pp. 5435-5448, 2018.

[18] M. S. A. Khan, S. Abdullah, A. Ali, F. Amin, and F. Hussain, "Pythagorean Hesitant fuzzy Choquet Integral aggregation operators and their application to group decision making," Soft Computing, vol. 23, pp. 251-267, 2019.

[19] H. Garg and D. Rani, "Multi-criteria decision making method based on Bonferroni mean aggregation operators of Complex intuitionistic fuzzy numbers," Journal of Industrial and Management Optimization, vol. 17, pp. 1-28, 2019.

[20] B. Batool, M. Ahmad, S. Abdullah, S. Ashraf, and R. Chinram, "Entropy based pythagorean probabilistic hesitant fuzzy decision making technique and its application for fog-haze factor Assessment problem," Entropy, vol. 22, no. 3, p. 318, 2020.

[21] H. Garg and D. Rani, "Some results on information measures for complex intuitionistic fuzzy sets," International Journal of Intelligent Systems, vol. 34, no. 10, pp. 2319-2363, 2019.

[22] M. J. Khan, M. I. Ali, and P. Kumam, "A new ranking technique for q-rung orthopair fuzzy values," International Journal of Intelligent Systems, vol. 36, no. 1, pp. 558-592, 2021.

[23] M. S. A. Khan, A. S. Khan, I. A. Khan, W. K. Mashwani, and F. Hussain, "Linguistic interval-valued Q-rung Orthopair fuzzy TOPSIS method for decision making problem with incomplete weight," Journal of Intelligent and Fuzzy Systems, vol. 40, no. 3, pp. 4223-4235.

[24] D. A. Molodtsov, "Soft set theory-first results," Computers and Mathematics with Applications, vol. 37, no. 4-5, pp. 19-31, 1999.

[25] K. M. Lee, "Bipolar valued fuzzy sets and their operations," in Proceedings of the International Conference on Intelligent Technologies, pp. 307-312, Bangkok, Tailand, December 2000.

[26] T. Mahmood, "A novel approach towards bipolar soft sets and their applications," Journal of Mathematics, vol. 2020, Article ID 4690808, 23 pages, 2020.

[27] D. Ramot, R. Milo, M. Friedman, and A. Kandel, "Complex fuzzy sets," IEEE Transactions on Fuzzy Systems, vol. 10, no. 2, pp. 171-186, 2002.

[28] D. Ramot, M. Friedman, G. Langholz, and A. Kandel, "Complex fuzzy logic," IEEE Transactions on Fuzzy Systems, vol. 11, no. 4, pp. 450-461, 2003.

[29] S. Dick, R. R. Yager, and O. Yazdanbakhsh, "On Pythagorean and complex fuzzy set operations," IEEE Transactions on Fuzzy Systems, vol. 24, no. 5, pp. 1009-1021, 2016.

[30] A Alkouri and A Salleh, "Complex intuitionistic fuzzy sets," Second International Conference on Fundamental and Applied Sciences, vol. 1482, pp. 464-470, 2012.

[31] A. Alkouri and A. Salleh, "Complex Atanassov's intuitionistic fuzzy relation," Abstract and Applied Analysis, vol. 2013, Article ID 287382, 18 pages, 2013.

[32] A. U. M. Alkouri and A. R. Salleh, "Some operations on complex Atanassov's intuitionistic fuzzy sets," AIP Conference Proceedings, vol. 1571, no. 1, pp. 987-993, 2013.

[33] D. Rani and H. Garg, "Complex intuitionistic fuzzy power aggregation operators and their applications in multicriteria decision-making," Expert Systems, vol. 35, no. 6, Article ID e12325, 2018.

[34] H. Garg and D. Rani, "Some generalized complex intuitionistic fuzzy aggregation operators and their application to multicriteria decision-making process," Arabian Journal for Science and Engineering, vol. 44, no. 3, pp. 2679-2698, 2019.

[35] Z. Xu, "An overview of methods for determining OWA weights," International Journal of Intelligent Systems, vol. 20, no. 8, pp. 843-865, 2005.

[36] H. Garg and D. Rani, "Exponential, logarithmic and compensative generalized aggregation operators under complex intuitionistic fuzzy environment, group decision and negotiation," Group Decision and Negotiation, vol. 28, no. 5, 2019.

[37] T. Kumar and R. K. Bajaj, "On complex intuitionistic fuzzy soft sets with distance measures and entropies," Journal of Mathematics, vol. 2014, Article ID 972198, 12 pages, 2014.

[38] F. Khan, M. S. A. Khan, M. Shahzad, and S. Abdullah, "Pythagorean cubic fuzzy aggregation operators and their application to multi-criteria decision making problems," Journal of Intelligent and Fuzzy Systems, vol. 36, no. 1, pp. 595-607, 2019. 